\begin{tabular}{|c|l|}
\hline Title & $\begin{array}{l}\text { Catalyst-free click cascade functionalization of unsaturated-bond-containing polymers using masked-ketene tethering } \\
\text { nitrile N-oxide }\end{array}$ \\
\hline Author(s) & Cheawchan, Sumitra; Koyama, Y asuhito; U chida, Satoshi; Takata, Toshikazu \\
\hline Citation & $\begin{array}{l}\text { Polymer, 54(17), 4501-4510 } \\
\text { https://doi.org/10.1016/.polymer.2013.06.020 }\end{array}$ \\
\hline Issue Date & 2013-08-02 \\
\hline Doc URL & http://hdl.handle.net/2115/53275 \\
\hline Type & article (author version) \\
\hline Additional Information & There are other files related to this item in HUSCAP. Check the above URL. \\
\hline File Information & Polymer20130606-1.pdf (Manuscript) \\
\hline
\end{tabular}

Instructions for use 


\section{Catalyst-Free Click Cascade Functionalization of Unsaturated-Bond-Containing Polymers Using Masked-Ketene-Tethering Nitrile $N$-Oxide}

Sumitra Cheawchan, ${ }^{a}$ Yasuhito Koyama, ${ }^{\mathrm{b}}$ * Satoshi Uchida, ${ }^{\mathrm{a}}$ and Toshikazu Takata ${ }^{\mathrm{a}, *}$


2-12-1-(H-126), Ookayama, Meguro, Tokyo 152-8552, Japan

${ }^{\mathrm{b}}$ Catalysis Research Center, Hokkaido University, N21 W10, Kita-ku, Sapporo 001-0021, Japan

* Corresponding author. Tel.: +81-11-706-9157, fax: +81-11-706-9156.

E-mail address: yasuhito.koyama@ cat.hokudai.ac.jp (Y. Koyama). 


\begin{abstract}
We developed a facile protocol for grafting-onto and cross-linking unsaturated-bond-containing common polymers via the formation of masked-ketene-functionalized polymers. The protocol utilizes a cascade functionalization agent $\mathbf{1}$ that has nitrile $N$-oxide and masked ketene functionalities. Through the model ligation reactions using $\mathbf{1}$, it turned out that the $\mathbf{1}$ facilitates the catalyst-free click introduction of masked ketene moieties to the unsaturated bonds such as $\mathrm{C} \equiv \mathrm{N}$ and $\mathrm{C}=\mathrm{C}$ bonds, capable of undergoing catalyst-free ligation with not only a nucleophilic amine but also a neutral alcohol with low nucleophilicity. On the basis of these results, the catalyst-free grafting reactions of PEG onto EPDM and PAN using 1 were performed to afford the corresponding graft copolymers in excellent conversion yields. In addition, it was also revealed that heating of both PAN and NR with masked ketene moieties at $250{ }^{\circ} \mathrm{C}$ for $1 \mathrm{~h}$ without catalyst enables the efficient conversion to give the respective cross-linked polymers.
\end{abstract}

Keywords: Nitrile $\mathrm{N}$-oxide; Cascade functionalization; Catalyst-free grafting onto and cross-linking reaction 


\section{Introduction}

Among various modification or functionalization methods of polymers, the stepwise protocol using a cascade functionalization tool is beneficial owing to the remarkable versatility of functional groups and a high functionalization ratio. However, it is inferior to direct methods in terms of the number of reactions employed. A masked-ketene-functionalized polymer (MKP) [1-10] is a potential reactive polymers that can be used for the post-modification of processed materials toward the development of advanced materials. The MKP skeleton includes ketene equivalents, such as Meldrum's acid derivatives and benzodioxinones, to generate a ketene functionality by external stimuli, e.g., heating [11,12] and photoirradiation [13]. The resulting ketene is highly reactive to various nucleophiles and provides the corresponding adducts without any catalyst [14-16]. The ketene gradually dimerizes in the absence of nucleophiles to give cyclobutadione [17]. Therefore, MKP exhibits dual reactivity to produce both polymers functionalized with nucleophiles and cross-linked polymers by self-reaction. In light of Yağci's [18-20] and Hawker's pioneering reports [21] of the synthesis of MKP based on the polymerization of masked-ketene-containing monomers for general materials applications, we developed a new protocol to directly form MKPs from common polymers using nitrile $N$-oxide agents.

Recently, we reported a new cascade functionalization agent for common polymers, consisting of an ambident agent having both nitrile $\mathrm{N}$-oxide and epoxide functionalities [22]. This agent enables the easy molecular integration of various nucleophiles onto $\mathrm{C}=\mathrm{C}$-, $\mathrm{C} \equiv \mathrm{C}$-, and $\mathrm{C} \equiv \mathrm{N}$-containing polymers in the presence of an appropriate catalyst. Building on our previous reports [23-32], in this study, we describe a convenient protocol to introduce a masked ketene moiety to 
unsaturated-bond-containing common polymers via a catalyst-free click reaction. Central to this method is the use of a new ambident agent that possesses both nitrile $N$-oxide and masked ketene functionalities (Fig. 1). Heating of the resulting MKPs enables both a catalyst-free grafting-onto reaction with a nucleophilic polymer and a catalyst-free cross-linking reaction.
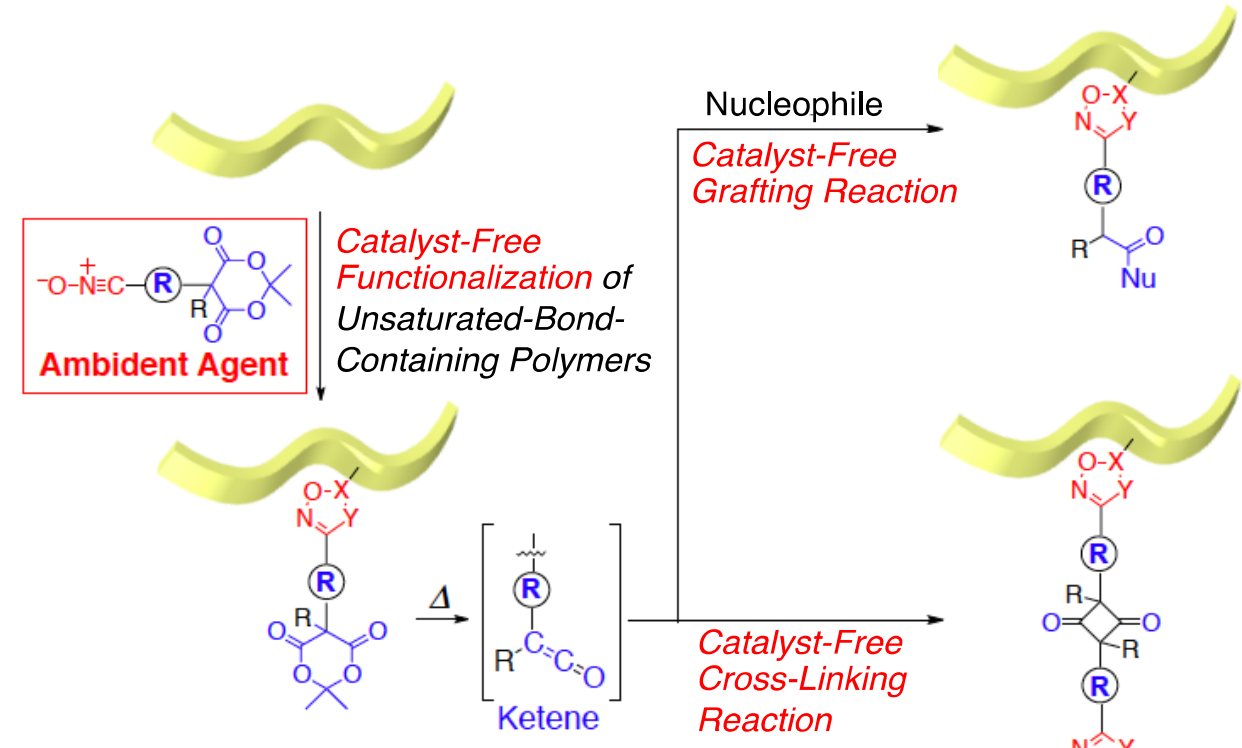

Fig. 1. Synthetic strategy for MKP and its subsequent modification to enable grafting and cross-linking using an ambident agent having nitrile $N$-oxide and masked ketene functionalities.

\section{Experimental section}

\subsection{Materials}

For NMR analyses, deuterated solvents with trimethylsilane by Across Organics Inc. were used. Wako Gel ${ }^{\circledR}$ C-400HG (Wako Chemical Inc.) was used for silica gel chromatography. Meldrum's acid was purchased from Wako Chemical Inc. All 
compounds given below bear the same formula numbers as used in the main body. Compounds unlabeled in the main body are labeled with letters $[\mathbf{A}-\mathbf{E}]$. Compound $\mathbf{A}$ was prepared according to the literature [33]. Other reagents and solvents commercially available were used without further purification unless otherwise noted.

\subsection{Characterization}

${ }^{1} \mathrm{H}$ NMR (400 MHz) and ${ }^{13} \mathrm{C}$ NMR (100 MHz) spectra were recorded on a JEOL AL-400 spectrometers using tetramethylsilane as an internal standard. MALDI-TOF MS spectra were measured with a Shimadzu AXIMA-CFR mass spectrometer using a dithranol matrix. Melting points were measured with a Stuart Scientific SMP3. SEC analyses were carried out on JASCO PU-2080 plus pump with a JASCO UV-1570 (UV detector) and JASCO RI-1530 (RI detector) equipped with a consecutive linear polystyrene gel columns TOSO TSK gel GMHXL and G5000HXL at $30{ }^{\circ} \mathrm{C}$. TGA analyses were carried out on a Shimadzu TGA-50 instrument at $\mathrm{N}_{2}$ atmosphere (flow rate of $50 \mathrm{~mL} / \mathrm{min})$ to determine $5 \%$ weight decomposition temperature $\left(T_{\mathrm{d} 5}\right)$ at which 5\% weight loss was observed. DSC analyses were carried out with a Shimadzu DSC-60 instrument at $\mathrm{N}_{2}$ atmosphere (flow rate of $50 \mathrm{~mL} / \mathrm{min}$ ) with liquid $\mathrm{N}_{2}$ as a refrigerant to determine glass transition temperature $\left(T_{\mathrm{g}}\right)$.

\subsection{Synthesis of new cascade fuctionalization agent 1}



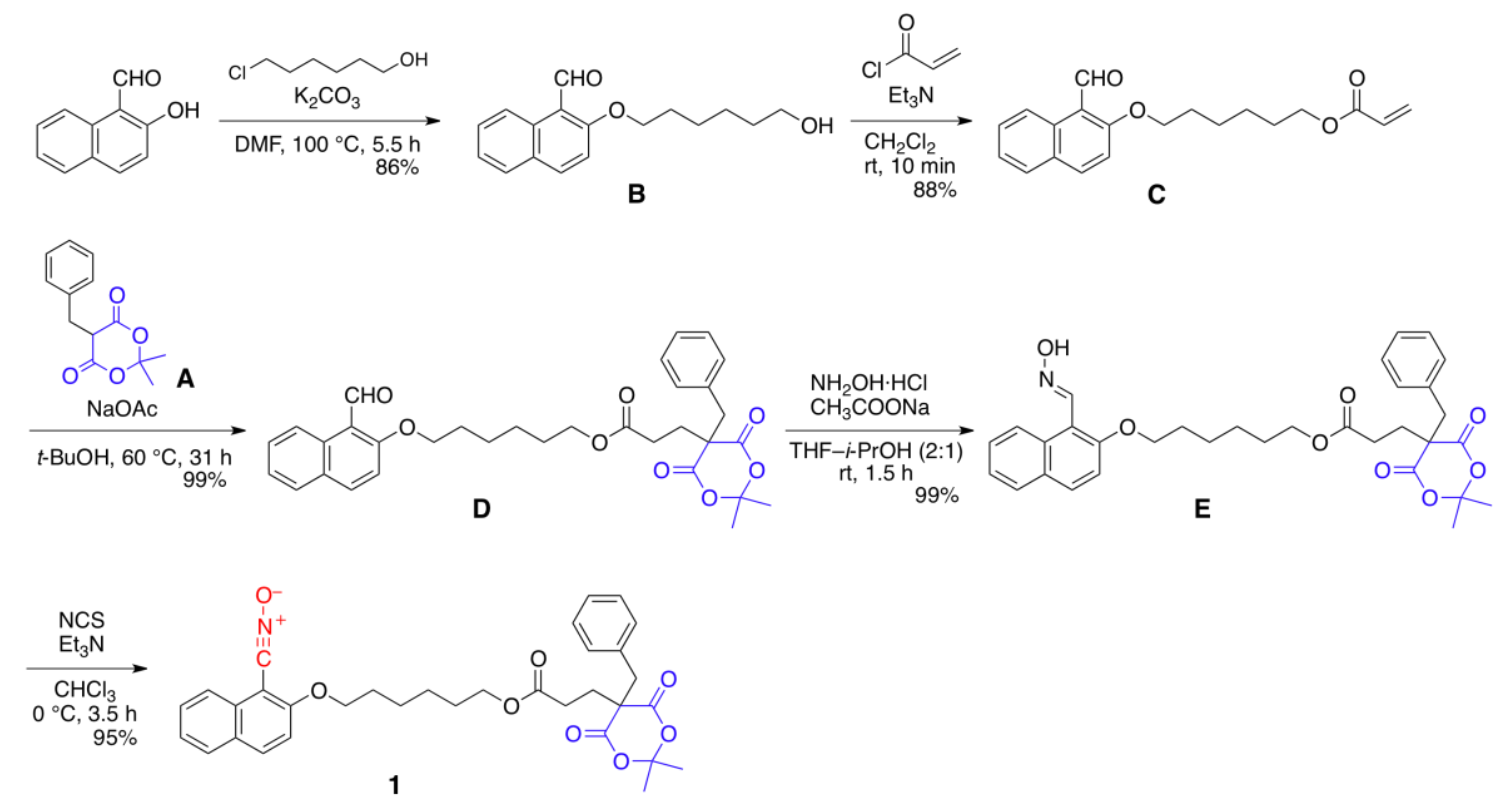

Scheme 1. Synthetic pathway of 1 .

\section{Synthesis of alcohol $\boldsymbol{B}$}

2-Hydroxy-1-naphthaldehyde (5.00 g, $29.0 \mathrm{mmol})$ was dissolved in DMF (146 mL) at room temperature. $\mathrm{K}_{2} \mathrm{CO}_{3}(4.81 \mathrm{~g}, 35.0 \mathrm{mmol})$ and 6-chlorohexanol (4.50 mL, 32.0 mmol) was added into the solution and the mixture was stirred at $100{ }^{\circ} \mathrm{C}$ for $5.5 \mathrm{~h}$, and cooled to room temperature. The mixture was diluted with $\mathrm{CH}_{2} \mathrm{Cl}_{2}$ and washed with water. The products were extracted with $\mathrm{CH}_{2} \mathrm{Cl}_{2}$ (200 mL, 3 times). The combined organic layer was washed with brine, dried over $\mathrm{MgSO}_{4}$, filtered, and concentrated in vacuo. The crude was purified by a flash column chromatography on silica gel (hexane: ethyl acetate $=1: 1)$ to afford the product $(\mathbf{B})$ as bright brown solids $(2.8 \mathrm{~g}$, 69\% yield); m.p. 81.1-83.4 ${ }^{\circ} \mathrm{C} ;{ }^{1} \mathrm{H}$ NMR (400 MHz, $\left.\mathrm{CDCl}_{3}, 298 \mathrm{~K}\right) \delta 10.93(\mathrm{~s}, 1 \mathrm{H})$, $9.28(\mathrm{~d}, J=8.6 \mathrm{~Hz}, 1 \mathrm{H}), 8.04(\mathrm{~d}, J=9.0 \mathrm{~Hz}, 1 \mathrm{H}), 7.77(\mathrm{~d}, J=8.0 \mathrm{~Hz}, 1 \mathrm{H}), 7.62(\mathrm{dd}, J=$ 8.6, 7.1 Hz, 1H), $7.42(\mathrm{dd}, J=8.0,7.1 \mathrm{~Hz}, 1 \mathrm{H}), 7.28(\mathrm{~d}, J=9.0 \mathrm{~Hz}, 1 \mathrm{H}), 4.24(\mathrm{t}, J=6.4$ $\mathrm{Hz}, 2 \mathrm{H}), 3.68(\mathrm{t}, J=6.4 \mathrm{~Hz}, 2 \mathrm{H}), 1.95-1.44(\mathrm{~m}, 8 \mathrm{H}) \mathrm{ppm} ;{ }^{13} \mathrm{C} \mathrm{NMR}\left(100 \mathrm{MHz}, \mathrm{CDCl}_{3}\right.$, 298 K) $\delta 192.2,163.5,137.5,131.5,128.4,128.2,124.9,124.7,119.0,116.2,113.5$, 
69.7, 62.8, 42.6, 39.3, 25.9, 25.5 ppm; IR (NaCl) v 3416 (O-H), 2936 (C-H(aromatic)), 2865 (C-H(aliphatic)), 2800 (C-H, aldehyde), 1669 (C=O(ether)), $1513\left(\mathrm{CH}_{2}\right.$ (alkane)), 1246 (C-O, as, Ar-O-R) cm ${ }^{-1}$; MALDI-TOF MS ( $/ \mathrm{z} / \mathrm{z}$ calc'd for $\mathrm{C}_{17} \mathrm{H}_{20} \mathrm{O}_{3} \mathrm{Na}[\mathrm{M}+\mathrm{Na}]^{+}$, 295.13; found, 294.49.

\section{Synthesis of acrylate $C$}

To a solution of alcohol $\mathbf{B}(3.00 \mathrm{~g}, 11 \mathrm{mmol})$ and acryloyl chloride $(1.80 \mathrm{~mL}, 22 \mathrm{mmol})$ in $\mathrm{CH}_{2} \mathrm{Cl}_{2}(15 \mathrm{~mL})$ was added dropwise $\mathrm{Et}_{3} \mathrm{~N}(3.06 \mathrm{~mL}, 22 \mathrm{mmol})$ by dropping at $0{ }^{\circ} \mathrm{C}$. The solution was stirred at room temperature for $10 \mathrm{~min}$. The reaction was quenched by the addition of sat. aq. $\mathrm{NaHCO}_{3}$. The products were extracted with $\mathrm{CH}_{2} \mathrm{Cl}_{2}$. The combined organic layer was dried over $\mathrm{MgSO}_{4}$, filtered, and concentrated in vacuo. The crude was purified by a flash column chromatography on silica gel (hexane: ethyl acetate $=1: 1)$ to give the product $\mathbf{C}$ as bright yellow solids $(2.37 \mathrm{~g}, 88 \%$ yield $)$; m.p. 41.8-42.9 ${ }^{\circ} \mathrm{C} ;{ }^{1} \mathrm{H} \mathrm{NMR}\left(400 \mathrm{MHz}, \mathrm{CDCl}_{3}, 298 \mathrm{~K}\right) \delta 10.93(\mathrm{~s}, 1 \mathrm{H}), 9.28(\mathrm{~d}, J=8.6 \mathrm{~Hz}$, 1H), $8.04(\mathrm{~d}, J=9.0 \mathrm{~Hz}, 1 \mathrm{H}), 7.77(\mathrm{~d}, J=8.0 \mathrm{~Hz}, 1 \mathrm{H}), 7.62(\mathrm{dd}, J=8.6,7.1 \mathrm{~Hz}, 1 \mathrm{H})$, $7.42(\mathrm{dd}, J=8.0,7.1 \mathrm{~Hz}, 1 \mathrm{H}), 7.28(\mathrm{~d}, J=9.0 \mathrm{~Hz}, 1 \mathrm{H}), 6.43(\mathrm{~d}, J=9.0 \mathrm{~Hz}, 1 \mathrm{H}), 6.38$ $(\mathrm{dd}, J=12.0 \mathrm{~Hz}, 1 \mathrm{H}), 5.82(\mathrm{~d}, J=9.0 \mathrm{~Hz}, 1 \mathrm{H}), 4.24-4.17(\mathrm{~m}, 4 \mathrm{H}), 1.93-1.48(\mathrm{~m}, 8 \mathrm{H})$ ppm; ${ }^{13} \mathrm{C}$ NMR $\left(100 \mathrm{MHz}, \mathrm{CDCl}_{3}, 298 \mathrm{~K}\right) \delta 192.6,166.8,164.0,138.1,132.0,131.1$, $130.3,129.3,129.0,127.3,125.4,117.1,117.1,113.9,69.8,64.9,29.7,29.0,28.2,26.2$ ppm; IR (NaCl) v 2940 (C-H(aromatic)), 2865 (C-H(aliphatic)), 2800 (C-H, aldehyde), $1730(\mathrm{C}=\mathrm{O}($ ester conjugated with $\mathrm{C}=\mathrm{C})), 1671 \quad(\mathrm{C}=\mathrm{O}$ (aldehyde conjugated with aromatic)), 1512 ( $\mathrm{CH}_{2}$ (alkane)), 1246-1153 (C-O, as, Ar-O-R) $\mathrm{cm}^{-1}$; MALDI-TOF MS $(m / z)$ calc'd for $\mathrm{C}_{20} \mathrm{H}_{22} \mathrm{O}_{4} \mathrm{Na}[\mathrm{M}+\mathrm{Na}]^{+}, 349.14$; found, 348.69 .

Synthesis of aldehyde $\boldsymbol{D}$ 
Compound C (2.41 g, $7.39 \mathrm{mmol})$ and Meldrum's acid derivative (A) (4.31 g, 18.4 mmol) was dissolved in $t$ - $\mathrm{BuOH}(15 \mathrm{~mL})$. Sodium acetate trihydrate $(5.03 \mathrm{~g}, 37.0$ mmol) was added to the mixture and the mixture was stirred at $60{ }^{\circ} \mathrm{C}$ for $31 \mathrm{~h}$. The mixture was cooled to room temperature, and poured into EtOAc. The solution was washed with water and the aqueous layer was extracted with EtOAc. The combined organic layer was washed with brine, dried over by $\mathrm{MgSO}_{4}$, filtered, and concentrated in vacuo. The crude was purified by a flash column chromatography on silica gel (hexane: $\mathrm{EtOAc}=5: 3$ ) to give $\mathbf{D}$ as bright yellow solids (4.12 g, 99\% yield); m.p. 107.0-107.5 ${ }^{\circ} \mathrm{C} ;{ }^{1} \mathrm{H}$ NMR (400 MHz, $\left.\mathrm{CDCl}_{3}, 298 \mathrm{~K}\right) \delta 10.93(\mathrm{~s}, 1 \mathrm{H}), 9.28(\mathrm{~d}, J=8.6$ $\mathrm{Hz}, 1 \mathrm{H}), 8.04(\mathrm{~d}, J=9.0 \mathrm{~Hz}, 1 \mathrm{H}), 7.77(\mathrm{~d}, J=8.0 \mathrm{~Hz}, 1 \mathrm{H}), 7.62(\mathrm{dd}, J=8.6,7.1 \mathrm{~Hz}$, 1H), $7.42(\mathrm{dd}, J=8.0,7.1 \mathrm{~Hz}, 1 \mathrm{H}), 7.30-7.17(\mathrm{~m}, 6 \mathrm{H}), 4.24(\mathrm{t}, J=6.3 \mathrm{~Hz}, 2 \mathrm{H}), 4.11(\mathrm{t}$, $J=6.8 \mathrm{~Hz}, 2 \mathrm{H}), 3.34(\mathrm{~s}, 2 \mathrm{H}), 2.50(\mathrm{t}, J=7.3 \mathrm{~Hz}, 2 \mathrm{H}), 2.38(\mathrm{t}, J=7.3 \mathrm{~Hz}, 2 \mathrm{H})$, 1.94-1.46 (m, 11H), 0.67 (s, 3H) ppm; ${ }^{13} \mathrm{C} \mathrm{NMR}\left(100 \mathrm{MHz}, \mathrm{CDCl}_{3}, 298 \mathrm{~K}\right) \delta 192.2$, $171.5,168.5,163.8,137.7,135.1,130.5,130.5,129.0,129.0,128.4,128.1,125.1,124.9$, $113.6,133.6,133.6,106.2,69.5,65.0,56.6,43.8,30.1,29.5,29.4,28.9,28.6,27.9,25.9$, 25.8 ppm; IR (NaCl) v 2941 (C-H(aromatic)), 2868 (C-H(aliphatic)), 2798 (C-H, aldehyde), 1770, $1733(\mathrm{C}=\mathrm{O}($ ester $)), 1673(\mathrm{C}=\mathrm{O}$ (aldehyde conjugated with aromatic) $)$, $1512\left(\mathrm{CH}_{2}\right.$ (alkane)), 1269-1155 (C-O, as, Ar-O-R) $\mathrm{cm}^{-1}$; MALDI-TOF MS ( $\left.\mathrm{m} / \mathrm{z}\right)$ calc'd for $\mathrm{C}_{33} \mathrm{H}_{36} \mathrm{O}_{8} \mathrm{Na}^{+}[\mathrm{M}+\mathrm{Na}]^{+}, 583.23$; found, 583.11.

\section{Synthesis of oxime $\boldsymbol{E}$}

Compound D (2.0 g, $3.57 \mathrm{mmol})$ was dissolved in a mixed solvent of THF and $i$-PrOH $(2: 1,30.6 \mathrm{~mL}) . \quad$ A solution of hydroxylammonium chloride $(281.1 \mathrm{mg}, 3.92 \mathrm{mmol})$ and sodium acetate trihydrate $(542.1 \mathrm{mg}, 3.92 \mathrm{mmol})$ in water $(5.1 \mathrm{~mL})$ was added dropwise into the solution of $\mathbf{D}$ at $0{ }^{\circ} \mathrm{C}$ during stirring for $30 \mathrm{~min}$. The mixture was 
warmed to room temperature and stirred for $1 \mathrm{~h}$. The mixture was diluted with EtOAc $(30 \mathrm{~mL})$, and washed with water three times. The organic layer was washed with brine, dried over $\mathrm{MgSO}_{4}$, filtered, and concentrated in vacuo to give $\mathbf{E}$ as yellow solids (2.03 g, $99 \%$ yield); ${ }^{1} \mathrm{H}$ NMR (400 MHz, $\left.\mathrm{CDCl}_{3}, 298 \mathrm{~K}\right) \delta 8.88(\mathrm{~s}, 1 \mathrm{H}), 8.77(\mathrm{~d}, J=8.8 \mathrm{~Hz}, 1 \mathrm{H})$, $7.85(\mathrm{~d}, J=9.0 \mathrm{~Hz}, 1 \mathrm{H}), 7.77(\mathrm{~d}, J=8.0 \mathrm{~Hz}, 1 \mathrm{H}), 7.51(\mathrm{dd}, J=8.8,7.1 \mathrm{~Hz}, 1 \mathrm{H}), 7.38$ $(\mathrm{dd}, J=8.0,7.1 \mathrm{~Hz}, 1 \mathrm{H}), 7.27-7.17(\mathrm{~m}, 6 \mathrm{H}), 4.17-4.09(\mathrm{~m}, 4 \mathrm{H}), 3.34(\mathrm{~s}, 2 \mathrm{H}), 2.49(\mathrm{t}, J$ $=8.9 \mathrm{~Hz}, 2 \mathrm{H}), 2.37(\mathrm{t}, J=8.9 \mathrm{~Hz}, 2 \mathrm{H}), 1.88-1.46(\mathrm{~m}, 11 \mathrm{H}), 0.65(\mathrm{~s}, 3 \mathrm{H}) \mathrm{ppm} ;{ }^{13} \mathrm{C}$ $\mathrm{NMR} \square\left(100 \mathrm{MHz}, \mathrm{CDCl}_{3}, 298 \mathrm{~K}\right) \delta 171.5,168.6,156.5,148.0,135.1,132.2,131.8$, $130.6,129.2,129.1,128.5,125.7,125.7,124.2,114.7,114.0,106.3,106.369 .6,65.1$, 56.6, 43.8, 30.2, 29.5, 29.4, 28.9, 28.6, 28.0, 26.0, 25.8 ppm; IR (NaCl) v $3445(\mathrm{O}-\mathrm{H})$, 2940 (C-H (aromatic)), 2868 (C-H (aliphatic)), 1770, $1733(\mathrm{C}=\mathrm{O}($ ester $)), 1652(\mathrm{C}=\mathrm{N})$, 1270 (C-O, as, Ar-O-R) $\mathrm{cm}^{-1}$; MALDI-TOF MS (m/z) calc'd for $\mathrm{C}_{33} \mathrm{H}_{37} \mathrm{NO}_{8} \mathrm{Na}^{+}$ $[\mathrm{M}+\mathrm{Na}]^{+}$, 598.24; found, 597.39 .

\section{Synthesis of cascade functionalization agent 1}

Compound $\mathbf{E}(2.03 \mathrm{~g}, 3.53 \mathrm{mmol})$ was dissolved in $\mathrm{CHCl}_{3}(35 \mathrm{~mL})$ at $0{ }^{\circ} \mathrm{C} . \mathrm{Et}_{3} \mathrm{~N}(690$ $\mu \mathrm{L}$ ) was added to the mixture. $\mathrm{N}$-Chlorosuccinimide $(577.9 \mathrm{mg}, 4.24 \mathrm{mmol})$ was portionwised into the solution, and the mixture was continually stirred at $0{ }^{\circ} \mathrm{C}$ for $3.5 \mathrm{~h}$. The reaction was quenched by pouring into water $(30 \mathrm{~mL})$. The products were extracted with $\mathrm{CHCl}_{3}$. The combined organic layer was washed with water 2 times, brine, dried over $\mathrm{MgSO}_{4}$, filtered, and concentrated in vacuo to obtain $\mathbf{1}$ as a bright yellow oil (1.92 g, 95\% yield); ${ }^{1} \mathrm{H}$ NMR (400 MHz, $\left.\mathrm{CDCl}_{3}, 298 \mathrm{~K}\right) \delta 7.98(\mathrm{~d}, J=8.8 \mathrm{~Hz}$, 1H), $7.93(\mathrm{~d}, J=9.0 \mathrm{~Hz}, 1 \mathrm{H}), 7.82(\mathrm{~d}, J=8.3 \mathrm{~Hz}, 1 \mathrm{H}), 7.60(\mathrm{dd}, J=8.9,7.1 \mathrm{~Hz}, 1 \mathrm{H})$, $7.43(\mathrm{dd}, J=8.3,7.0 \mathrm{~Hz}, 1 \mathrm{H}), 7.27-7.17(\mathrm{~m}, 6 \mathrm{H}), 4.22(\mathrm{t}, J=6.6 \mathrm{~Hz}, 2 \mathrm{H}), 4.11(\mathrm{t}, J=$ $6.8 \mathrm{~Hz}, 2 \mathrm{H}), 3.34(\mathrm{~s}, 2 \mathrm{H}), 2.50(\mathrm{t}, J=8.8 \mathrm{~Hz}, 2 \mathrm{H}), 2.38(\mathrm{t}, J=8.1 \mathrm{~Hz}, 2 \mathrm{H}), 1.93-1.47(\mathrm{~m}$, 
11H), $0.67(\mathrm{~s}, 3 \mathrm{H}) \mathrm{ppm} ;{ }^{13} \mathrm{C} \mathrm{NMR}\left(100 \mathrm{MHz}, \mathrm{CDCl}_{3}, 298 \mathrm{~K}\right) \delta 171.5,168.6,161.0$, $135.2,132.9,130.6,130.6,129.1,128.9,128.5,128.1,128.1,125.1,124.1,113.4,106.2$, $106.2,69.6,65.1,56.6,43.8,30.2,29.5,29.2,28.9,28.6,28.6,25.8,25.8$ ppm; IR $(\mathrm{NaCl}) \cup 2941$ (C-H(aromatic), 2868 (C-H(aliphatic)), 2292 (C $\equiv \mathrm{N}), 1771,1739$ $(\mathrm{C}=\mathrm{O}($ ester $)), 1274$ (C-O, as, Ar-O-R $) \mathrm{cm}^{-1}$; MALDI-TOF MS $(\mathrm{m} / \mathrm{z})$ calc'd for $\mathrm{C}_{33} \mathrm{H}_{35} \mathrm{NO}_{8} \mathrm{Na}^{+}[\mathrm{M}+\mathrm{Na}]^{+}, 596.23$; found, 595.38.

2.4. Synthesis of model compounds obtained by using cascade functionalization agent 1<smiles>CC1(C)OC(=O)C(CCC(=O)OCCCCCCOc2ccc3ccccc3c2C#[N+][O-])(Cc2ccccc2)C(=O)O1</smiles>
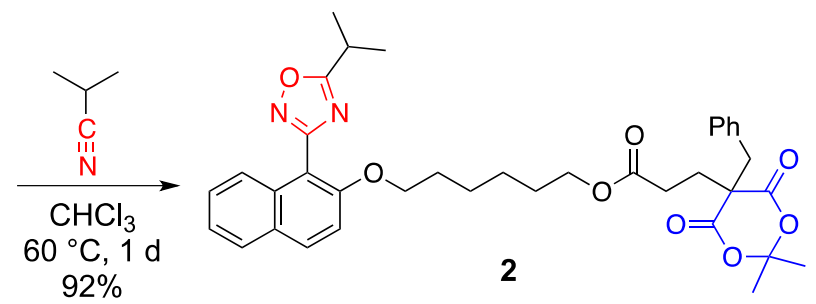

Free of Catalyst


3

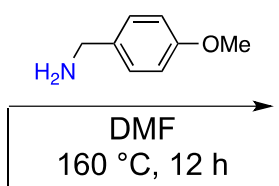

$85 \%$

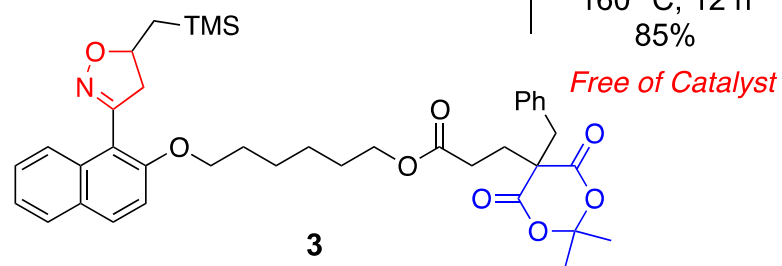

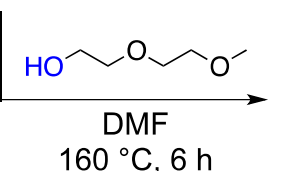
$60 \%$ Free of Catalyst

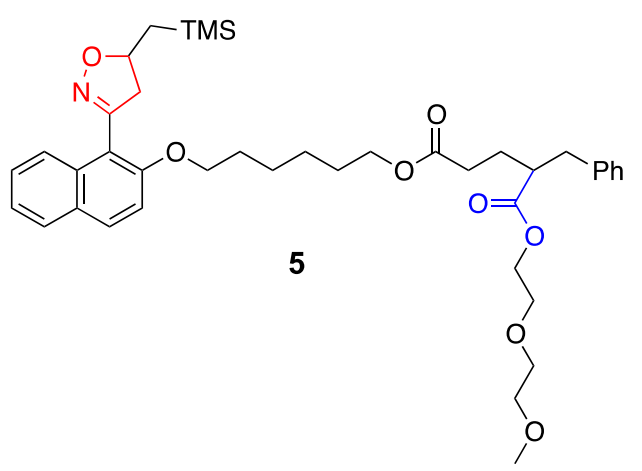

Scheme 2. Catalyst-free ligation between unsaturated bond and nucleophile using $\mathbf{1}$. 
Compound 1 (50 mg, $87.2 \mu \mathrm{mol})$ was dissolved in $\mathrm{CHCl}_{3}(0.5 \mathrm{~mL})$. Isobutyronitrile (78.3 $\mu \mathrm{L}, 872 \mu \mathrm{mol})$ was added to the mixture. The mixture was stirred at $60^{\circ} \mathrm{C}$ for 1 d, cooled to room temperature, and concentrated in vacuo to give 2 (56.0 $\mathrm{mg}, 92 \%$ yield) as an yellow oil; ${ }^{1} \mathrm{H}$ NMR $\left(400 \mathrm{MHz}, \mathrm{CDCl}_{3}, 298 \mathrm{~K}\right) \delta 7.95(\mathrm{~d}, J=9.2 \mathrm{~Hz}, 1 \mathrm{H})$, $7.81(\mathrm{~d}, J=8.1 \mathrm{~Hz}, 1 \mathrm{H}), 7.62(\mathrm{~d}, J=8.6 \mathrm{~Hz}, 1 \mathrm{H}), 7.44(\mathrm{dd}, J=8.1,7.0 \mathrm{~Hz}, 1 \mathrm{H})$, 7.39-7.17 (m, 7H), $4.13(\mathrm{t}, J=6.5 \mathrm{~Hz}, 2 \mathrm{H}), 4.06(\mathrm{t}, J=6.5 \mathrm{~Hz}, 2 \mathrm{H}), 3.41-3.34(\mathrm{~m}, 1 \mathrm{H})$, $3.34(\mathrm{~s}, 2 \mathrm{H}), 2.49(\mathrm{t}, J=8.7 \mathrm{~Hz}, 2 \mathrm{H}), 2.36(\mathrm{t}, J=8.7 \mathrm{~Hz}, 2 \mathrm{H}), 1.75-1.36(\mathrm{~m}, 17 \mathrm{H}), 0.65$ (s, 3H) ppm; $\left.{ }^{13} \mathrm{C} \mathrm{NMR} \mathrm{(100} \mathrm{MHz,} \mathrm{CDCl}_{3}, 298 \mathrm{~K}\right) \delta 183.6,171.3,168.4,165.4,155.9$, $135.0,133.2,132.2,132.2,130.4,128.9,128.7,128.0,127.5,127.4,124.1,114.4,110.8$ $106.1,69.5,65.0,56.5,43.6,35.3,30.0,30.0,29.4,29.1,28.8,28.5,27.6,25.6,20.3$ ppm; IR (NaCl) v 2938 (C-H(aromatic)), 2867 (C-H(aliphatic)), 1772, 1738 $(\mathrm{C}=\mathrm{O}($ ester $)), 1271$ (C-O, as, Ar-O-R), $1624(\mathrm{C}=\mathrm{N}) \mathrm{cm}^{-1}$; MALDI-TOF MS $(\mathrm{m} / \mathrm{z})$ calc'd for $\mathrm{C}_{37} \mathrm{H}_{42} \mathrm{~N}_{2} \mathrm{O}_{8} \mathrm{Na}^{+}[\mathrm{M}+\mathrm{Na}]^{+}, 665.28$; found, 665.41.

\section{Synthesis of isoxazoline 3}

Compound 1 (50 mg, $87.2 \mu \mathrm{mol})$ was dissolved in $\mathrm{CHCl}_{3}(0.5 \mathrm{~mL})$. Allytrimethysilane $(141.3 \mu \mathrm{L})$ was added to the mixture. The mixture was stirred at $60{ }^{\circ} \mathrm{C}$ for $1 \mathrm{~d}$, cooled to room temperature, and concentrated in vacuo to give $\mathbf{3}$ as a mixture of the target product (3) (59.3 mg, 90\% yield) and the regio-isomer (10\%). $\quad 3:{ }^{1} \mathrm{H}$ NMR (400 MHz, $\left.\mathrm{CDCl}_{3}, 298 \mathrm{~K}\right) \delta 8.04(\mathrm{~d}, J=8.8 \mathrm{~Hz}, 1 \mathrm{H}), 7.87(\mathrm{~d}, J=9.0 \mathrm{~Hz}, 1 \mathrm{H}), 7.78(\mathrm{~d}, J=8.0 \mathrm{~Hz}$ 1H), $7.48(\mathrm{dd}, J=8.8,7.3 \mathrm{~Hz}, 1 \mathrm{H}), 7.43(\mathrm{dd}, J=8.0,7.3 \mathrm{~Hz}, 1 \mathrm{H}), 7.27-7.17(\mathrm{~m}, 6 \mathrm{H})$, 5.29-4.87 (m, 1H), 4.16-4.07 (m, 4H), $3.42(\mathrm{dd}, J=9.4,9.3 \mathrm{~Hz}, 2 \mathrm{H}), 3.33$ (s, 2H), 2.99 (dd, $J=9.4,9.3 \mathrm{~Hz}, 2 \mathrm{H}), 2.49$ (t, $J=7.2 \mathrm{~Hz}, 2 \mathrm{H}), 2.37$ (t, $J=7.2 \mathrm{~Hz}, 2 \mathrm{H}), 1.86-1.39$ (m, 12H), $1.14(\mathrm{dd}, J=8.9,8.8 \mathrm{~Hz}, 1 \mathrm{H}), 0.67(\mathrm{~s}, 3 \mathrm{H}), 0.12$ (s, 9H) ppm; ${ }^{13} \mathrm{C}$ NMR (100 $\left.\mathrm{MHz}, \mathrm{CDCl}_{3}, 298 \mathrm{~K}\right) \delta 172.2,169.4,156.2,155.8,135.9,133.6,132.1,131.4,131.4$, 
$129.9,129.9,128.9,128.3,125.4,124.9,114.8,114.7,107.1,80.6,70.1,65.9,57.4,47.2$, 44.6, 36.2, 30.3, 29.7, 26.8, 26.8, 26.7, 26.7, 25.1, 25.1, 0.0 ppm; IR (NaCl) $v 2940$ (C-H(aromatic)), 2859 (C-H(aliphatic)), 1769, 1742 (C=O(ester)), 1270 (C-O, as, Ar-O-R) $\mathrm{cm}^{-1}$; MALDI-TOF MS $(\mathrm{m} / z)$ calc'd for $\mathrm{C}_{39} \mathrm{H}_{49} \mathrm{NO}_{8} \mathrm{SiNa}^{+}[\mathrm{M}+\mathrm{Na}]^{+}, 710.31$; found; $T_{\mathrm{d} 5} 248^{\circ} \mathrm{C}$.

\section{Synthesis of 4}

Isoxazoline $\mathbf{3}(40 \quad \mathrm{mg}, \quad 58.2 \mu \mathrm{mol})$ was dissolved in $\operatorname{DMF}(116 \mu \mathrm{L})$. 4-methoxybenzylamine $(76.0 \mu \mathrm{L}, 582 \mu \mathrm{mol})$ was added to the mixture. The mixture was refluxed for $12 \mathrm{~h}$, cooled to room temperature, and concentrated in vacuo. The crude was purified by a flash column chromatography on silica gel $\left(\mathrm{CH}_{2} \mathrm{Cl}_{2}: \mathrm{MeOH}=\right.$ $10: 1)$ in to give 4 as a bright yellow oil $\left(35.7 \mathrm{mg}, 85 \%\right.$ yield); ${ }^{1} \mathrm{H}$ NMR (400 MHz, $\left.\mathrm{CDCl}_{3}, 298 \mathrm{~K}\right) \delta 8.02(\mathrm{~d}, J=8.6 \mathrm{~Hz}, 1 \mathrm{H}), 7.87(\mathrm{~d}, J=9.0 \mathrm{~Hz}, 1 \mathrm{H}), 7.78(\mathrm{~d}, J=8.2 \mathrm{~Hz}$, 1H), $7.47(\mathrm{dd}, J=7.0,8.6 \mathrm{~Hz}, 1 \mathrm{H}), 7.36(\mathrm{dd}, J=7.0,8.2 \mathrm{~Hz}, 1 \mathrm{H}), 7.27-7.13(\mathrm{~m}, 6 \mathrm{H})$, $6.87(\mathrm{~d}, J=8.8 \mathrm{~Hz}, 2 \mathrm{H}), 6.74(\mathrm{~d}, J=8.8 \mathrm{~Hz}, 2 \mathrm{H}), 4.93-4.88(\mathrm{~m}, 1 \mathrm{H}), 4.33(\mathrm{~d}, J=6.2$ $\mathrm{Hz}, 2 \mathrm{H}), 4.30(\mathrm{~d}, J=6.2 \mathrm{~Hz}, 2 \mathrm{H}), 4.13(\mathrm{t}, J=6.7 \mathrm{~Hz}, 2 \mathrm{H}), 4.05(\mathrm{t}, J=4.2 \mathrm{~Hz}, 2 \mathrm{H}), 3.77$ (s, 3H), 3.41 (dddd, $J=2.5,2.5,2.4,2.4 \mathrm{~Hz}, 1 \mathrm{H}), 3.01-2.90(\mathrm{~m}, 3 \mathrm{H}), 2.75(\mathrm{~d}, J=6.2$ $\mathrm{Hz}, 2 \mathrm{H}), 2.72(\mathrm{~d}, J=6.2 \mathrm{~Hz}, 2 \mathrm{H}), 2.42-2.36(\mathrm{~m}, 2 \mathrm{H}), 2.32-2.23(\mathrm{~m}, 1 \mathrm{H}), 2.06-2.02(\mathrm{~m}$, $2 \mathrm{H}), 1.84-0.86(\mathrm{~m}, 10 \mathrm{H}), 0.11(\mathrm{~s}, 3 \mathrm{H}), \mathrm{ppm} ;{ }^{13} \mathrm{C} \mathrm{NMR}\left(100 \mathrm{MHz}, \mathrm{CDCl}_{3}, 298 \mathrm{~K}\right) \quad \delta$ $174.7,174.3,154.4,137.7,137.0,135.0,132.1,130.1,130.0,129.9,129.9,129.4,129.4$, $129.0,128.3,127.3,125.4,125.0,114.8,114.8,80.6,70.2,65.4,56.5,54.4,50.0,47.0$, 43.8, 40.1, 32.9, 30.7, 30.4, 29.5, 28.9, 26.8, 25.1, 0.0 ppm; IR (NaCl) $v 3300(\mathrm{~N}-\mathrm{H})$, 2931 (C-H(aromatic)), 2849 (C-H(aliphatic)), 1733 (C=O(ester)), 1646 (C=O(amide)), 1248 (C-O) $\mathrm{cm}^{-1}$; MALDI-TOF MS $(\mathrm{m} / \mathrm{z})$ calc'd for $\mathrm{C}_{43} \mathrm{H}_{54} \mathrm{~N}_{2} \mathrm{O}_{6} \mathrm{SiNa}^{+}[\mathrm{M}+\mathrm{Na}]^{+}$, 745.37; found, 745.29. 


\section{Synthesis of 5}

Isoxazoline 3 (25 mg, $36.4 \mu \mathrm{mol})$ was dissolved in DMF (374 $\mu \mathrm{L})$. Diethylene glycol monomethyl ether $(42.8 \mu \mathrm{L}, 364 \mu \mathrm{mol})$ was added to the mixture. The mixture was refluxed for $8 \mathrm{~h}$, cooled to room temperature, and concentrated in vacuo. The crude was purified by a flash column chromatography on silica gel $\left(\mathrm{CH}_{2} \mathrm{Cl}_{2}\right)$ in to give $\mathbf{5}$ as a bright yellow oil (15.4 mg, 60\% yield); ${ }^{1} \mathrm{H}$ NMR (400 MHz, $\left.\mathrm{CDCl}_{3}, 298 \mathrm{~K}\right) \delta 8.04$ (d, $J$ $=8.3 \mathrm{~Hz}, 1 \mathrm{H}), 7.87(\mathrm{~d}, J=9.0 \mathrm{~Hz}, 1 \mathrm{H}), 7.78(\mathrm{~d}, J=8.3 \mathrm{~Hz}, 1 \mathrm{H}), 7.48(\mathrm{dd}, J=8.3,7.3$ $\mathrm{Hz}, 1 \mathrm{H}), 7.36(\mathrm{dd}, J=8.3,7.3 \mathrm{~Hz}, 1 \mathrm{H}), 7.26-7.15(\mathrm{~m}, 6 \mathrm{H}), 4.95-4.87(\mathrm{~m}, 1 \mathrm{H}), 4.18(\mathrm{t}, J$ $=4.6 \mathrm{~Hz}, 2 \mathrm{H}), 4.13(\mathrm{t}, J=6.4 \mathrm{~Hz}, 2 \mathrm{H}), 4.06(\mathrm{t}, J=6.6 \mathrm{~Hz}, 2 \mathrm{H}), 3.59-3.50(\mathrm{~m}, 9 \mathrm{H}), 3.36$ (s, 3H), 3.01-2.95 (m, 1H), $2.78(\mathrm{t}, J=6.1 \mathrm{~Hz}, 2 \mathrm{H}), 2.38-2.30(\mathrm{~m}, 1 \mathrm{H}), 1.85-0.88(\mathrm{~m}$, 12H), 0.12 (s, 9H), ppm; ${ }^{13} \mathrm{C}$ NMR (100 MHz, $\left.\mathrm{CDCl}_{3}, 298 \mathrm{~K}\right) \delta 175.7,173.9,156.2$, $155.8,139.8,133.6,132.0,129.9,129.9,129.4,129.0,128.3,127.4,125.4,125.0,114.8$, $114.5,80.6,72.8,71.4,70.2,70.0,65.4,64.4,60.1,47.6,47.0,39.3,32.8,30.7,30.7$, 30.4, 29.6, 27.8, 26.8, 0.0 ppm; IR (NaCl) $v 2924$ (C-H(aromatic)), 2849 (C-H(aliphatic)), 1733 (C=O(ester)), 1248 (C-O, as, Ar-O-R) $\mathrm{cm}^{-1}$; MALDI-TOF MS $(m / z)$ calc'd for $\mathrm{C}_{40} \mathrm{H}_{55} \mathrm{NO}_{8} \mathrm{SiNa}^{+}[\mathrm{M}+\mathrm{Na}]^{+}, 728.36$; found, 728.18.

\subsection{Typical procedure for polymer modification using $\mathbf{1}$}

Typical procedure for modification of PAN with 1 under solution reaction (Table 1, entry 2)

A solution of PAN $\left(M_{\mathrm{w}} 150,000\right)(50 \mathrm{mg}, 943.4 \mu \mathrm{mol})$ and $1(54.0 \mathrm{mg}, 94.2 \mu \mathrm{mol})$ in DMF $(2.0 \mathrm{~mL})$ was stirred at $60{ }^{\circ} \mathrm{C}$ for $3 \mathrm{~d}$. The mixture was cooled to room temperature, and precipitated into $\mathrm{H}_{2} \mathrm{O}$, washed again with $\mathrm{CHCl}_{3}$. The precipitates were collected by filtration and dried in vacuo to give the Meldrum's 
acid-functionalized PAN (99\% yield, 35\% conversion) as a white solid; ${ }^{1} \mathrm{H}$ NMR (400 MHz, DMSO, 298 K) $\delta 8.03-7.03$ (m), 4.12-4.39 (m), 3.32 (m), 3.25-3.13 (m), 2.65 (s), 2.44-2.02 (m), 1.67-1.23 (m), 0.70 (s) ppm; IR (KBr) v $2243(\mathrm{C} \equiv \mathrm{N}), 1735$ $(\mathrm{C}=\mathrm{O}($ ester $)), 1453(\mathrm{H}-\mathrm{C} \equiv \mathrm{N}), 1270(\mathrm{C}-\mathrm{O}$, as, Ar-O-R $) \mathrm{cm}^{-1} ; T_{\mathrm{d} 5} 287^{\circ} \mathrm{C} ; T_{\mathrm{g}} 105^{\circ} \mathrm{C}$.

Typical procedure for modification of NR with $\mathbf{1}$ under solution reaction (entry 4) $\mathrm{NR}\left(M_{\mathrm{w}} 1,250,000\right)(50 \mathrm{mg}, 0.735 \mathrm{mmol})$ was dissolved in $\mathrm{CHCl}_{3}(0.5 \mathrm{~mL})$ overnight. Compound 1 ( $42.1 \mathrm{mg}, 74 \mu \mathrm{mol})$ was added to the mixture. The solution was stirred at $60{ }^{\circ} \mathrm{C}$ for $3 \mathrm{~d}$. The solution was cooled to room temperature, and poured into $\mathrm{MeOH}$ to give precipitates. The precipitates were collected by filtration and dried in vacuo to give the Meldrum's acid-functionalized NR (99\% yield, $16 \%$ conversion); ${ }^{1} \mathrm{H}$ NMR $\left(400 \mathrm{MHz}, \mathrm{CDCl}_{3}, 298 \mathrm{~K}\right) \delta 8.08-7.19(\mathrm{~m}), 5.12(\mathrm{~s}), 4.22-4.11(\mathrm{~m}), 3.34(\mathrm{~s}), 2.47-1.25$ (m), $0.66(\mathrm{~s})$ ppm; IR (NaCl) $v 1737\left(\mathrm{C}=\mathrm{O}\right.$ (ester), $1270\left(\mathrm{C}-\mathrm{O}\right.$, as, Ar-O-R) cm ${ }^{-1} ; T_{\mathrm{d} 5}$ $213^{\circ} \mathrm{C} ; T_{\mathrm{g}}-23^{\circ} \mathrm{C}$.

Typical procedure for modification of $N R$ with 1 under solid-state reaction (entry 7) $\mathrm{NR}\left(M_{\mathrm{w}} 1,250,000\right)(50 \mathrm{mg}, 735 \mu \mathrm{mol})$ and $1(42.1 \mathrm{mg}, 74 \mu \mathrm{mol})$ were ground in a mortar at $70^{\circ} \mathrm{C}$ for $2 \mathrm{~h}$. The mixture was cooled to room temperature, and dissolved with $\mathrm{CHCl}_{3}$, and poured into $\mathrm{MeOH}$ to give precipitates. The precipitates were collected by filtration and dried in vacuo to give the Meldrum's acid-functionalized NR (99\% yield, 37\% conversion) as a yellow solid; ${ }^{1} \mathrm{H}$ NMR $\left(400 \mathrm{MHz}, \mathrm{CDCl}_{3}, 298 \mathrm{~K}\right) \delta$ 8.08-7.19 (m), $5.12(\mathrm{~s}), 4.22-4.11(\mathrm{~m}), 3.34(\mathrm{~s}), 2.47-1.25(\mathrm{~m}), 0.66$ (s) ppm; IR (KBr) v $1737(\mathrm{C}=\mathrm{O}($ ester $)), 1270(\mathrm{C}-\mathrm{O}$, as, Ar-O-R $) \mathrm{cm}^{-1} ; T_{\mathrm{d} 5} 213^{\circ} \mathrm{C} ; T_{\mathrm{g}}-23^{\circ} \mathrm{C}$.

Typical procedure for modification of NBR with 1 under solid-state reaction (entry 8) 
NBR (acrylonitrile: 33\%, $50 \mathrm{mg}, 930.7 \mu \mathrm{mol})$ and $1(53.4 \mathrm{mg}, 93.7 \mu \mathrm{mol})$ were ground in a mortar at $70{ }^{\circ} \mathrm{C}$ for $2 \mathrm{~h}$. The mixture was cooled to room temperature, and dissolved with $\mathrm{CHCl}_{3}$, and poured into $\mathrm{MeOH}$ to give precipitates. The precipitates were collected by filtration and dried in vacuo to give the Meldrum's acid-functionalized NBR (99\% yield, $85 \%$ conversion) as an yellow solid; ${ }^{1} \mathrm{H}$ NMR (400 MHz, $\left.\mathrm{CDCl}_{3}, 298 \mathrm{~K}\right) \delta$ 7.97-7.18 (m), 5.54-5.41 (m), 5.11-4.97 (m), 4.15-4.09 (m), 3.66-3.49 (m), $3.33(\mathrm{~s}), 2.59-1.16(\mathrm{~m}), 0.66(\mathrm{~s})$ ppm; IR $(\mathrm{KBr}) \cup 2235(\mathrm{C} \equiv \mathrm{N})$, $1739(\mathrm{C}=\mathrm{O}($ ester $)), 1267(\mathrm{C}-\mathrm{O}$, as, $\mathrm{Ar}-\mathrm{O}-\mathrm{R}) \mathrm{cm}^{-1} ; T_{\mathrm{d} 5} 332^{\circ} \mathrm{C} ; T_{\mathrm{g}}-3.4^{\circ} \mathrm{C}$.

Typical procedure for modification of EPDM with 1 under solid-state reaction (entry 9) EPDM (ENB: 10\%, $50 \mathrm{mg}, 115 \mu \mathrm{mol})$ and $1(38.2 \mathrm{mg}, 115 \mu \mathrm{mol})$ were ground in a mortar at $70{ }^{\circ} \mathrm{C}$ for $2 \mathrm{~h}$. The mixture was cooled to room temperature, and dissolved with $\mathrm{CHCl}_{3}$, and poured into $\mathrm{MeOH}$ to give precipitates. The precipitates were collected by filtration and dried in vacuo to give the Meldrum's acid-functionalized EPDM (99\% yield, 64\% conversion) as an yellow rubber; ${ }^{1} \mathrm{H}$ NMR (400 $\mathrm{MHz}, \mathrm{CDCl}_{3}$, 298 K) $\delta 8.07-7.17(\mathrm{~m}), 5.25-5.02(\mathrm{~m}), 4.22-4.11(\mathrm{~m}), 3.34(\mathrm{~s}), 2.47-0.83(\mathrm{~m}), 0.66(\mathrm{~s})$ ppm; IR (KBr) $v 1735(\mathrm{C}=\mathrm{O}($ ester $)), 1261$ (C-O, as, Ar-O-R) $\mathrm{cm}^{-1} ; T_{\mathrm{d} 5} 309^{\circ} \mathrm{C} ; T_{\mathrm{g}}$ $-17^{\circ} \mathrm{C}$

\subsection{Typical procedure for grafting-onto reaction}

Typical procedure for grafting reaction of PEG onto EPDM

Meldrum's acid-functionalized EPDM (25 mg, $19.8 \mu \mathrm{mol}, 64 \%$ conversion) was dissolved in mesitylene $(632 \mu \mathrm{L})$. To the solution was added MeOPEGOH $\left(M_{\mathrm{n}} 350\right)$ (63.4 $\mu \mathrm{L}, 19 \mu \mathrm{mol})$ and $\mathrm{CaSO}_{4}(63.2 \mu \mathrm{mg})$. The mixture was stirred at room temperature for $1 \mathrm{~h}$ and heated at $160{ }^{\circ} \mathrm{C}$ for $9 \mathrm{~h}$. The solution was cooled to room 
temperature, and poured into water to give precipitates. The precipitates were collected by filtration, and the filtrate was washed with $\mathrm{H}_{2} \mathrm{O}$ and $\mathrm{MeOH}$ and dried in vacuo to give the PEG-grafted EPDM (99\% yield, 98\% conversion of Meldrum's acid); ${ }^{1} \mathrm{H}$ NMR (400 MHz, $\left.\mathrm{CDCl}_{3}, 298 \mathrm{~K}\right) \delta$ 8.14-6.95 (m), 5.25-5.02 (m), 4.34-4.23 (m), 3.92-3.46 (m), 2.45-0.88 (m) ppm; IR (KBr) v 1260 (C-O, as, Ar-O-R), $\mathrm{cm}^{-1} ; T_{\mathrm{d} 5}$ $217^{\circ} \mathrm{C} ; T_{\mathrm{g}}-18^{\circ} \mathrm{C}$.

Typical procedure for one-pot grafting reaction of PEG onto PAN

A mixture of PAN (50 mg, $943.4 \mu \mathrm{mol}), 1$ (54.1 mg, $94.3 \mu \mathrm{mol})$, MeOPEGOH ( $M_{\mathrm{n}} 350$, $330.2 \mu \mathrm{L}, 943.4 \mu \mathrm{mol})$, and $\mathrm{CaSO}_{4}(100 \mathrm{mg})$ in DMF $(1.0 \mathrm{~mL})$ was stirred at room temperature for $1 \mathrm{~h}$. The mixture was warmed to $60{ }^{\circ} \mathrm{C}$ and stirred for $1 \mathrm{~d}$. The reaction temperature was elevated to $160{ }^{\circ} \mathrm{C}$ and stirred for $12 \mathrm{~h}$. The mixture was cooled to room temperature, filtered, and poured into water to give precipitates. The precipitates were collected by filtration and the filtrate was washed with $\mathrm{H}_{2} \mathrm{O}$ and $\mathrm{CHCl}_{3}$ and dried in vacuo to give the PEG-grafted PAN (249.2 mg, 99\% yield, 27\% conversion for the 1,3-dipolar cycloaddition, 97\% conversion for the grafting reaction); ${ }^{1} \mathrm{H}$ NMR (400 MHz, DMSO, $\left.298 \mathrm{~K}\right) \delta$ 8.31-7.17 (m), 4.12-4.03 (m), 3.59 (s), 3.48-3.12 (m), $2.75(\mathrm{~s}), 2.44-2.02(\mathrm{~m}), 1.67-1.23(\mathrm{~m}) \mathrm{ppm} ; \mathrm{IR}(\mathrm{KBr}) \cup 2243(\mathrm{C} \equiv \mathrm{N})$, $1600(\mathrm{C}=\mathrm{O}($ ester $)), 1453(\mathrm{H}-\mathrm{C} \equiv \mathrm{N}), 1270\left(\mathrm{C}-\mathrm{O}\right.$, as, Ar-O-R $\mathrm{cm}^{-1} ; T_{\mathrm{d} 5} 292{ }^{\circ} \mathrm{C} ; T_{\mathrm{g}}$ $133{ }^{\circ} \mathrm{C}$.

2.7. Typical procedure for cross-linking reaction of Meldrum's acid-functionalized polymer: Synthesis of cross-linked NR

NR with $16 \%$ Meldrum's acid functionality $(15.0 \mathrm{mg}, 0.1 \mathrm{mmol})$ was dissolved in $\mathrm{CHCl}_{3}$. The mixture was placed to Teflon plate and heated at $40{ }^{\circ} \mathrm{C}$ to give a 
self-standing film. The film was heated at $250{ }^{\circ} \mathrm{C}$ for $1 \mathrm{~h}$ under $\mathrm{N}_{2}$ to give the cross-linked polymer. The cross-linked polymer was purified by swelling in toluene to remove the unreacted materials, and gently dried at room temperature for $1 \mathrm{~d}$ to give the corresponding cross-linked NR $(11.4 \mathrm{mg})$. To evaluate the cross-linking efficiency, the cross-linked polymer was immersed into toluene overnight to give the swollen gel (swelling ratio: $1,300 \%)$. The cross-linking density $\left(\mathrm{v}, \mathrm{mol} / \mathrm{cm}^{3}\right)$ was calculated by the modified Flory-Rehner equation [34] to evaluate that the interchain reaction efficiency of Meldrum's acid moieties on the cross-linking reaction was 25\%: By the following Flory-Rehner equation, the cross-linking density $(\mathrm{v})$ was calculated to be $1.31 \times 10^{-5}$ $\mathrm{mol} / \mathrm{cm}^{3}$.

$v=-\frac{g}{V}\left[\frac{\ln \left(1-V_{R}\right)+V_{R}+\mu V_{R}^{2}}{g^{2 / 3} V_{R}^{2 / 3}-V_{R} / 2}\right]$

, where $\mathrm{v}, \mathrm{V}, \mathrm{g}, \mu$, and $\mathrm{V}_{\mathrm{R}}$ are cross-linking density $\left(\mathrm{mol} / \mathrm{cm}^{3}\right)$, molar volume of the solvent (toluene: $106.3 \mathrm{~cm}^{3} / \mathrm{mol}$ ), volume fraction of cross-linked gel (1.003), interaction parameter between polymer and solvent (natural rubber-toluene: 0.39 ), and volume fraction of polymer in the gel $\left(6.19 \times 10^{-2}\right)$, respectively. Therefore, the interchain reaction efficiency of Meldrum's acid moieties on the cross-linking reaction would be described with the following equation:

$$
\begin{aligned}
& \text { interchain reaction efficiency }(\%) \\
& =\frac{\text { [observed cross-linking density } \left.\left.\left(\mathrm{mol} / \mathrm{cm}^{3}\right)\right] \times \text { [sample volume }\left(\mathrm{cm}^{3}\right)\right]}{\text { [expected mol of cross-linking points as } 100 \% \text { efficiency }(\mathrm{mol})]} \times \text { [yield] }
\end{aligned}
$$

\section{Results and discussion}

\subsection{Model study of 1}

Before investigating the utility of $\mathbf{1}$ as an attachment tool for common polymers, 
we performed model ligation reactions to evaluate its reactivity toward the 1,3-dipolar cycloaddition reaction of $\mathrm{C}=\mathrm{C}$ and $\mathrm{C} \equiv \mathrm{N}$ groups and the subsequent nucleophilic substitution to the thermally generated ketene moiety, as shown in Scheme 2.

The treatment of $\mathbf{1}$ with isobutyronitrile or allyltrimethylsilane at $60{ }^{\circ} \mathrm{C}$ without a catalyst produced the corresponding heteroaromatics $\mathbf{2}$ and $\mathbf{3}$ in high yields, indicating adequate reactivity of the nitrile $N$-oxide moiety of $\mathbf{1}$ to $\mathrm{C} \equiv \mathrm{N}$ and $\mathrm{C}=\mathrm{C}$ bonds. The subsequent catalyst-free nucleophilic attack at the thermally generated ketene of $\mathbf{3}$ was examined using an amine and an alcohol. Since the gradual weight loss of $\mathbf{3}$ (attributed to the elimination of acetone and $\mathrm{CO}_{2}$ from the masked ketene moiety [35]) was observed at around $160{ }^{\circ} \mathrm{C}$ by thermogravimetric analysis (TGA), we conducted the nucleophilic modification at this temperature. As a result, refluxing a mixture of $\mathbf{3}$ and the nucleophiles in DMF produced the corresponding adducts $\mathbf{4}$ and $\mathbf{5}$ in high yields without a catalyst. It should be noted that $\mathbf{1}$ enables catalyst-free ligation between the unsaturated bond and not only a nucleophilic amine but also a neutral alcohol with low nucleophilicity. Figure 2 shows ${ }^{1} \mathrm{H}$ NMR spectra of (A) 1, (B) 3, and (C) 5. In the spectrum (B), the appearance of the signals originating from the isoxazoline skeleton protons and TMS protons afforded the direct evidence for the formation of $\mathbf{3}$. In the spectrum $(\mathrm{C})$, the characteristic signals of diethylene glycol monomethyl ether $(\alpha, \beta, \gamma$, $\delta$, and $\mathrm{OMe}$ ) along with the disappearance of signals of geminal dimethyl protons of Meldrum's acid moiety support the formation of 5. The ratio of ${ }^{1} \mathrm{H}$ NMR integrals of TMS protons, the isoxazoline protons, and the protons from the alcohol as a nucleophile clearly indicates that the cascade functionalization agent $\mathbf{1}$ served as a molecular glue to mediate successfully the ligation reaction between allyltrimethylsilane and diethylene glycol monomethyl ether. 


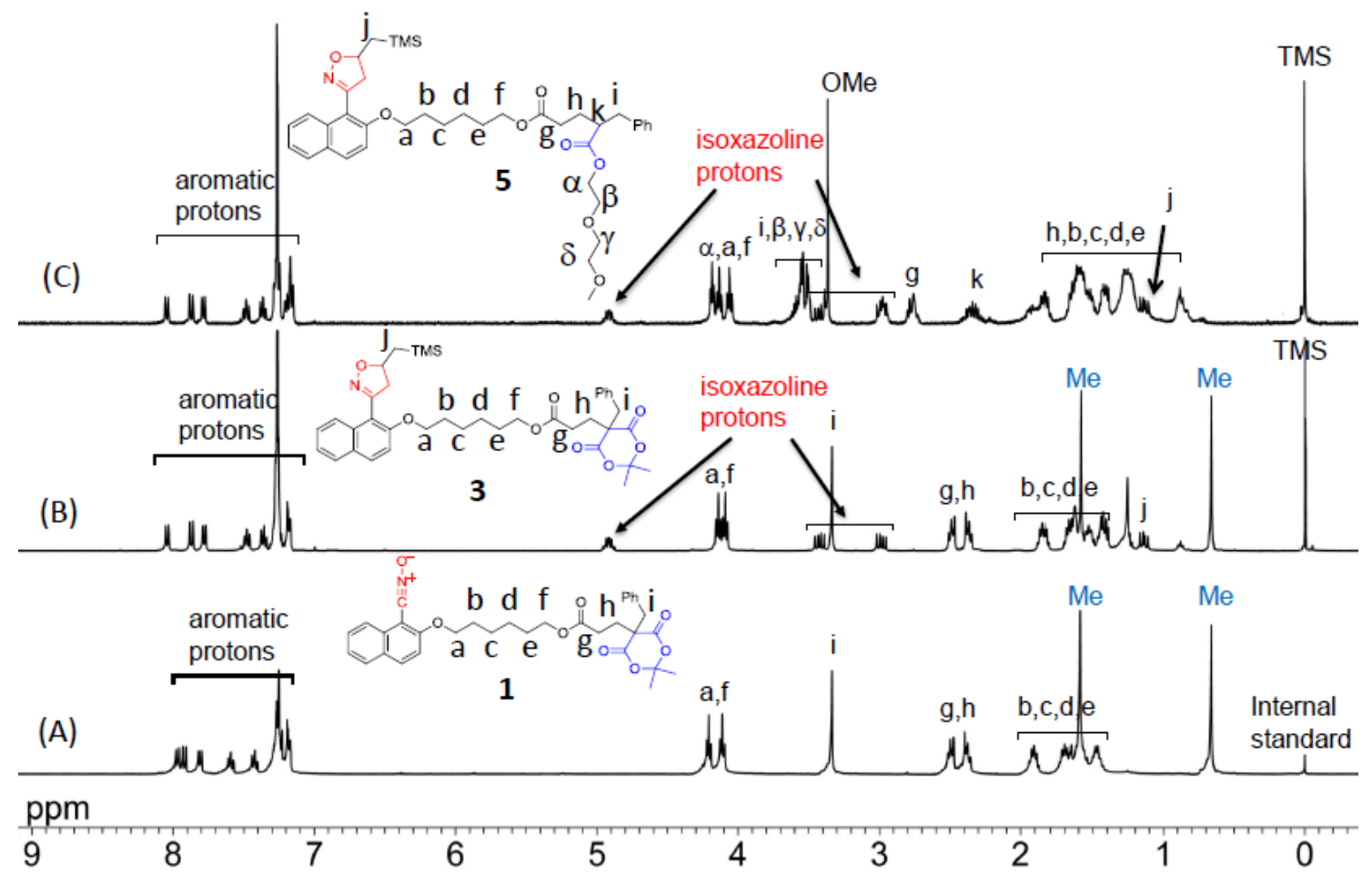

Fig. 2. ${ }^{1} \mathrm{H}$ NMR spectra of (A) 1, (B) 3, and (C) 5 (400 MHz, $\left.\mathrm{CDCl}_{3}, 298 \mathrm{~K}\right)$.

\subsection{Synthesis of functionalized polymers}

On the basis of these results, we next investigated the reactions of various unsaturated-bond-containing polymers with $1 . \quad$ The results are summarized in Table 1. 
Table 1. Functionalization of various polymers with $\mathbf{1}$.

\begin{tabular}{|c|c|c|c|c|c|c|}
\hline Entry & Polymer & $\begin{array}{l}\text { Reaction } \\
\text { Media }\end{array}$ & $\begin{array}{l}\text { Temp. } \\
\left({ }^{\circ} \mathrm{C}\right)\end{array}$ & $\begin{array}{l}\text { Time } \\
\text { (h) }\end{array}$ & $\begin{array}{l}\text { Conversion for } \\
\text { Cycloaddition }^{\mathrm{a}} \\
(\%)\end{array}$ & $\begin{array}{l}\text { Functionalization } \\
\text { Ratio of Polymer } \\
(\%)\end{array}$ \\
\hline $1^{\mathrm{b}}$ & PAN & $\mathrm{DMF}$ & 60 & 24 & 25 & 2.5 \\
\hline $2^{\mathrm{b}}$ & PAN & $\mathrm{DMF}$ & 60 & 72 & 35 & 3.5 \\
\hline $3^{b}$ & NR & $\mathrm{CHCl}_{3}$ & 60 & 24 & 12 & 1.2 \\
\hline $4^{b}$ & NR & $\mathrm{CHCl}_{3}$ & 60 & 72 & 16 & 1.6 \\
\hline $5^{\mathrm{b}}$ & NR & $-^{\mathrm{c}}$ & $\mathrm{rt}$ & 2 & 21 & 2.1 \\
\hline $6^{\mathrm{b}}$ & NR & $-^{\mathrm{c}}$ & $\mathrm{rt}$ & 72 & 59 & 5.9 \\
\hline $7^{\mathrm{b}}$ & NR & $-^{\mathrm{c}}$ & 70 & 2 & 37 & 3.7 \\
\hline $8^{\mathrm{b}}$ & NBR & $-^{\mathrm{c}}$ & 70 & 2 & $\begin{array}{l}\text { Olefin: } 90 \text {, } \\
\text { CN: } 76\end{array}$ & $\begin{array}{l}\text { Olefin: } 9.0 \text {, } \\
\text { CN: } 7.6\end{array}$ \\
\hline $9^{d}$ & EPDM & $-^{\mathrm{c}}$ & 70 & 2 & 64 & 64 \\
\hline
\end{tabular}

${ }^{a}$ Estimated by ${ }^{1} \mathrm{H}$ NMR. ${ }^{\mathrm{b}}$ Reaction was performed by using 0.1 equiv of $1 .{ }^{\mathrm{c}}$ Reaction was performed in the solid state. ${ }^{\mathrm{d}} 1.0$ equiv of 1 was used.

The reaction in DMF using 0.1 equiv of 1 per repeating unit of polyacrylonitrile (PAN) at $60{ }^{\circ} \mathrm{C}$ for $24 \mathrm{~h}$ resulted in the formation of oxadiazole-containing polymer (Table 1, entries 1 and 2). Increasing the reaction time increased the conversion percentage for the cycloaddition reaction but only up to a maximum of $35 \%$ (entry 2 ). The cycloaddition of $\mathbf{1}$ to natural rubber (NR) in $\mathrm{CHCl}_{3}$ gave the corresponding functionalized NR in low conversion yields (entries 3 and 4), probably because of the low reactivity (caused by steric hindrance) of the trisubstituted internal olefin in NR [22]. Through considerable effort, it was found that the solvent-free press-grinding of 
a mixture of NR and $\mathbf{1}$ enabled a more rapid cycloaddition reaction with higher conversion of NR $(21 \%)$ than in the solution reactions (entries 3 and 4), even when the reaction is performed at room temperature (entry 5).

In addition, a prolonged reaction time at room temperature or an elevated reaction temperature $\left(70{ }^{\circ} \mathrm{C}\right.$ ) increased the conversion yields (entry 6: 59\%, entry 7 : 37\%). The reactions of two commercially available elastomers, acrylonitrile-butadiene rubber (NBR) and ethylene-propylene-diene terpolymer (EPDM), gave the corresponding modified polymers in high conversion yields (entries 8 and 9). Such high conversion yields are probably attributed to the higher reactivity of the disubstituted olefin in NBR and the exo-olefin in EPDM to the nitrile $N$-oxide of 1 in comparison with the trisubstituted olefin in NR.

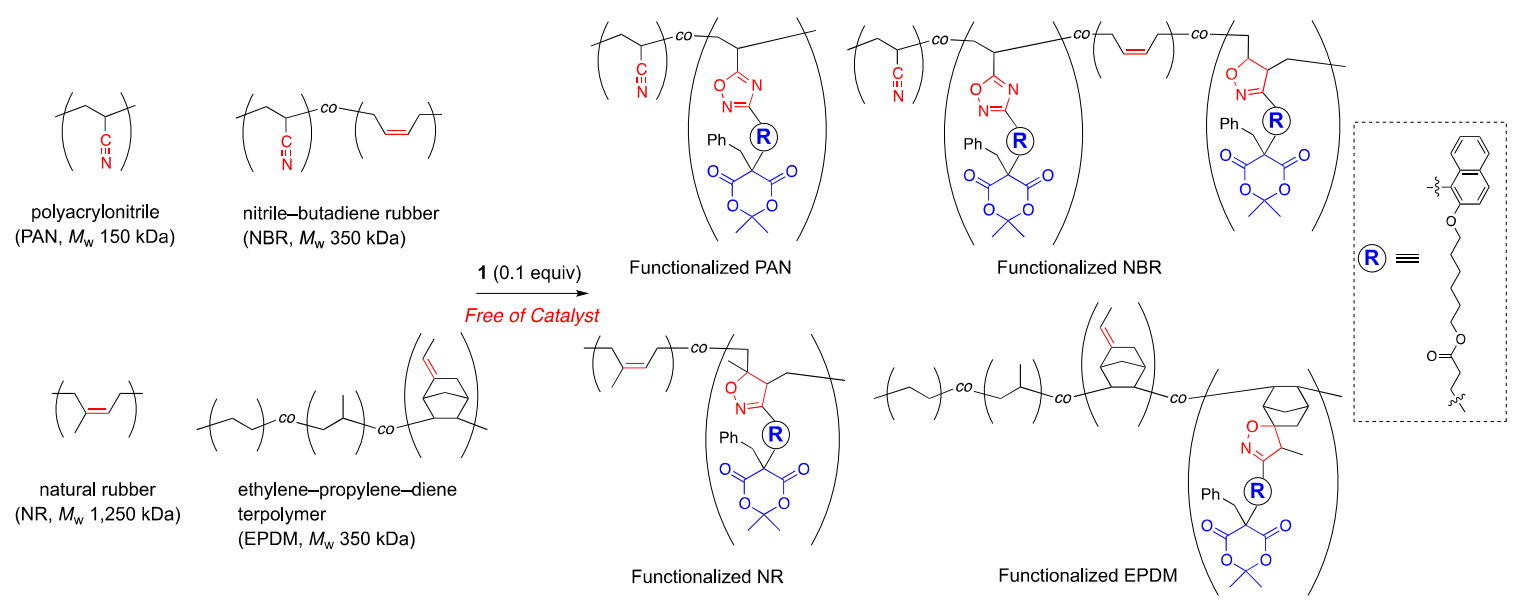

Scheme 3. Catalyst-free functionalization of unsaturated-bond-containing polymers using 1.

Having produced several masked-ketene-containing polymers, we demonstrated the grafting reaction using monomethyl-terminated polyethylene glycol (Scheme 4). We attempted the treatment of masked-ketene-functionalized EPDM (64\% conversion) 
with 10 equiv of PEG in mesitylene at $160{ }^{\circ} \mathrm{C}$ for $9 \mathrm{~h}$. The corresponding PEG-grafted EPDM was obtained with $50 \%$ grafting conversion. On the other hand, when $\mathrm{CaSO}_{4}$ was added to the reaction mixture as a dehydrating agent, the grafting-onto reaction produced PEG-grafted EPDM in an excellent conversion yield (98\%).

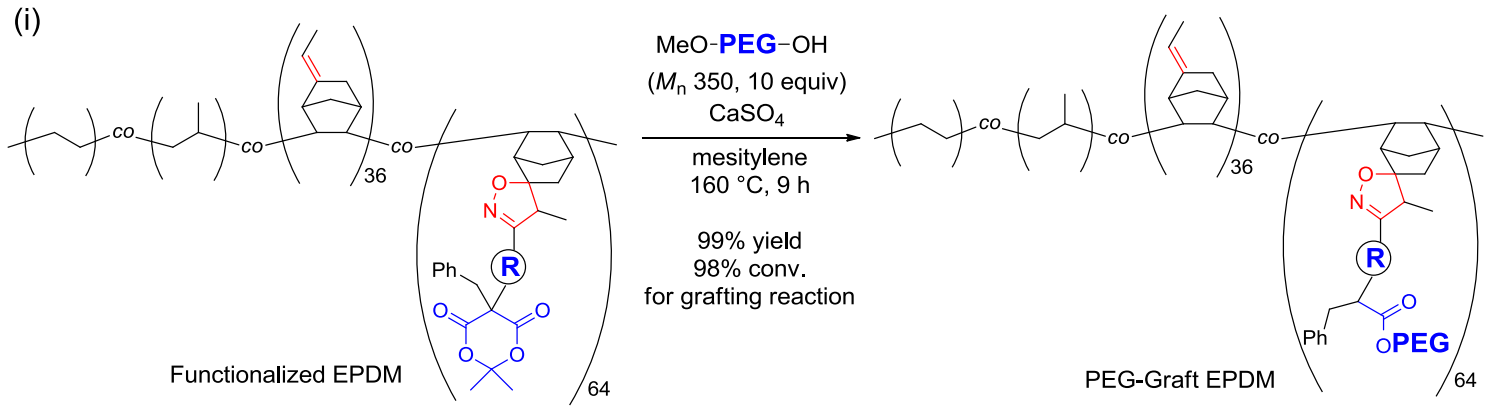

(ii)

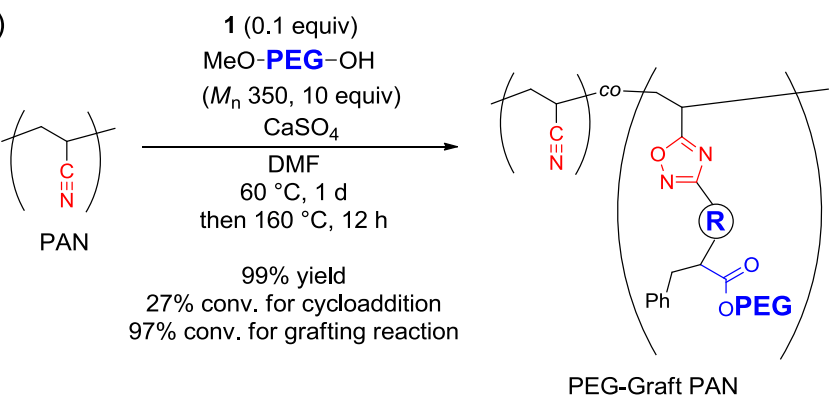

Scheme 4. (i) Catalyst-free grafting of PEG ( $\left.M_{\mathrm{n}} \quad 350\right)$ onto masked-ketene-functionalized EPDM and (ii) one-pot grafting of PEG onto PAN.

The structure of PEG-grafted EPDM was determined by ${ }^{1} \mathrm{H}$ NMR, IR spectral analysis, and SEC. Figure 3 shows ${ }^{1} \mathrm{H}$ NMR spectra of (a) PAN, (b) functionalized PAN with 3\% functionalization ratio, and (c) PEG-grafted PAN. In the spectrum (c), the signals originating from PAN, the skeleton of $\mathbf{1}$, and PEG along with disappearance of the protons attributed to the Meldrum's acid moieties are in good accordance with the formation of PEG-grafted PAN. From the integral ratio between the protons from $\mathbf{1}$ and the main chain protons of PAN, we determined that the conversion for 
cycloaddition reaction was $27 \%$. In addition, we determined the conversion for grafting reaction of PEG was 97\%, which was calculated from the integral ratio between the protons from 1 and the protons of PEG considering the number average molecular weight of PEG $\left(M_{\mathrm{n}} 350\right)$. It is noted that the conversion for the cycloaddition during the one-pot grafting reaction is in a good agreement with that in the absence of MeO-PEG-OH (Table 1, entry 1, 25\%), emphasizing that the 1,3-dipolar cycloaddition of nitrile $N$-oxide and nucleophilic substitution of PEG to the thermally generated ketene are not competitive.

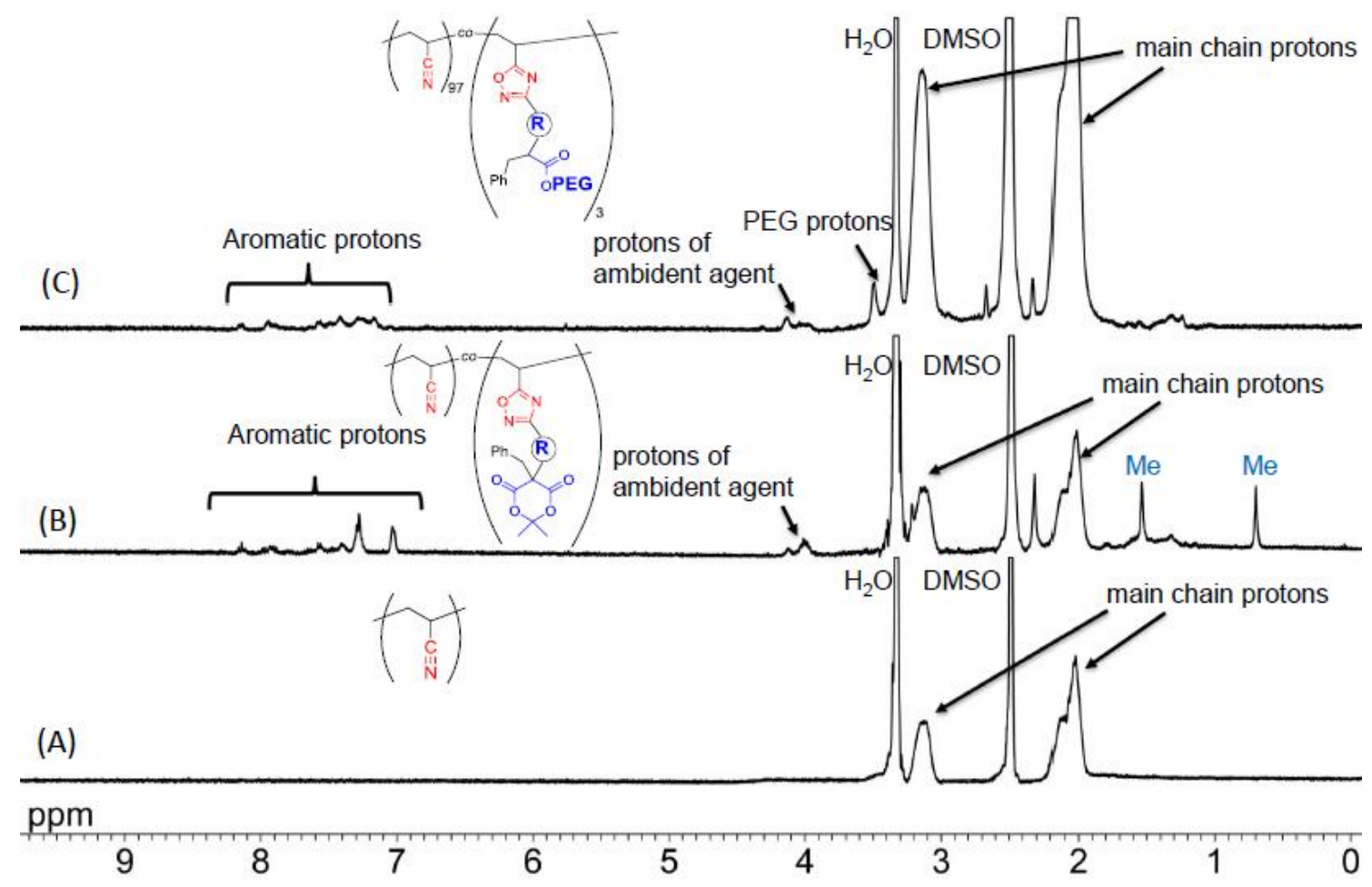

Fig. 3. ${ }^{1} \mathrm{H}$ NMR spectra of (a) PAN, (b) functionalized PAN with $3 \%$ functionalization ratio, and (c) PEG-grafted PAN (400 MHz, DMSO- $d_{6}, 298 \mathrm{~K}$ ).

The SEC profiles of EPDM and PEG-grafted EPDM are shown in Fig. 4. PEG-grafted EPDM appeared as a monomodal peak at an elution time later than that of 
unreacted EPDM. This result indicates a distinct structural change in EPDM, and it is consistent with a stronger interaction between the polar graft chains on the polymer and the SEC stationary phase.

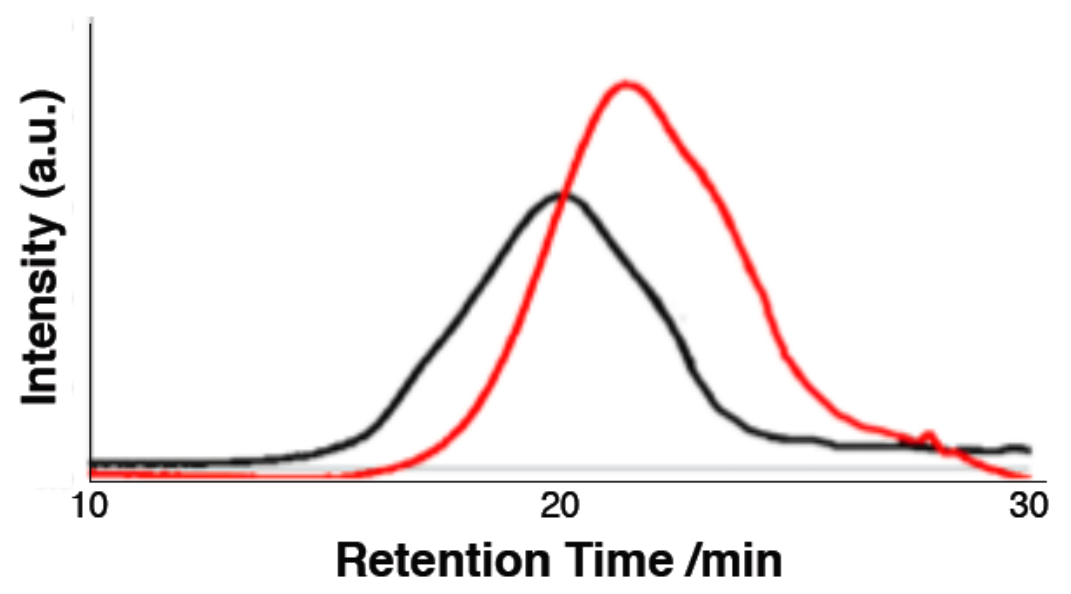

Fig. 4. SEC profiles of EPDM (black line) and PEG-grafted EPDM (red line) (eluent: DMF, $303 \mathrm{~K})$.

In addition, we examined a one-pot grafting reaction of PEG onto PAN. The step-by-step heating of a mixture of PAN, 0.1 equiv of 1 , and PEG $\left(60{ }^{\circ} \mathrm{C}, 1 \mathrm{~d}\right.$ then $160{ }^{\circ} \mathrm{C}$ ) without a catalyst was performed and the latter process at $160{ }^{\circ} \mathrm{C}$ was monitored by using the SEC profiles (Fig. 5). As a result, we found that the SEC change finished in $12 \mathrm{~h}$, indicating the completion of the addition reaction of PEG to the polymer backbone. The longer reaction time of grafting reaction than that from $\mathbf{3}$ to $\mathbf{5}$ ( $6 \mathrm{~h}$, Scheme 1) could be probably attributed to the steric repulsion between PEG as a nucleophile and PAN as a trunk polymer. 


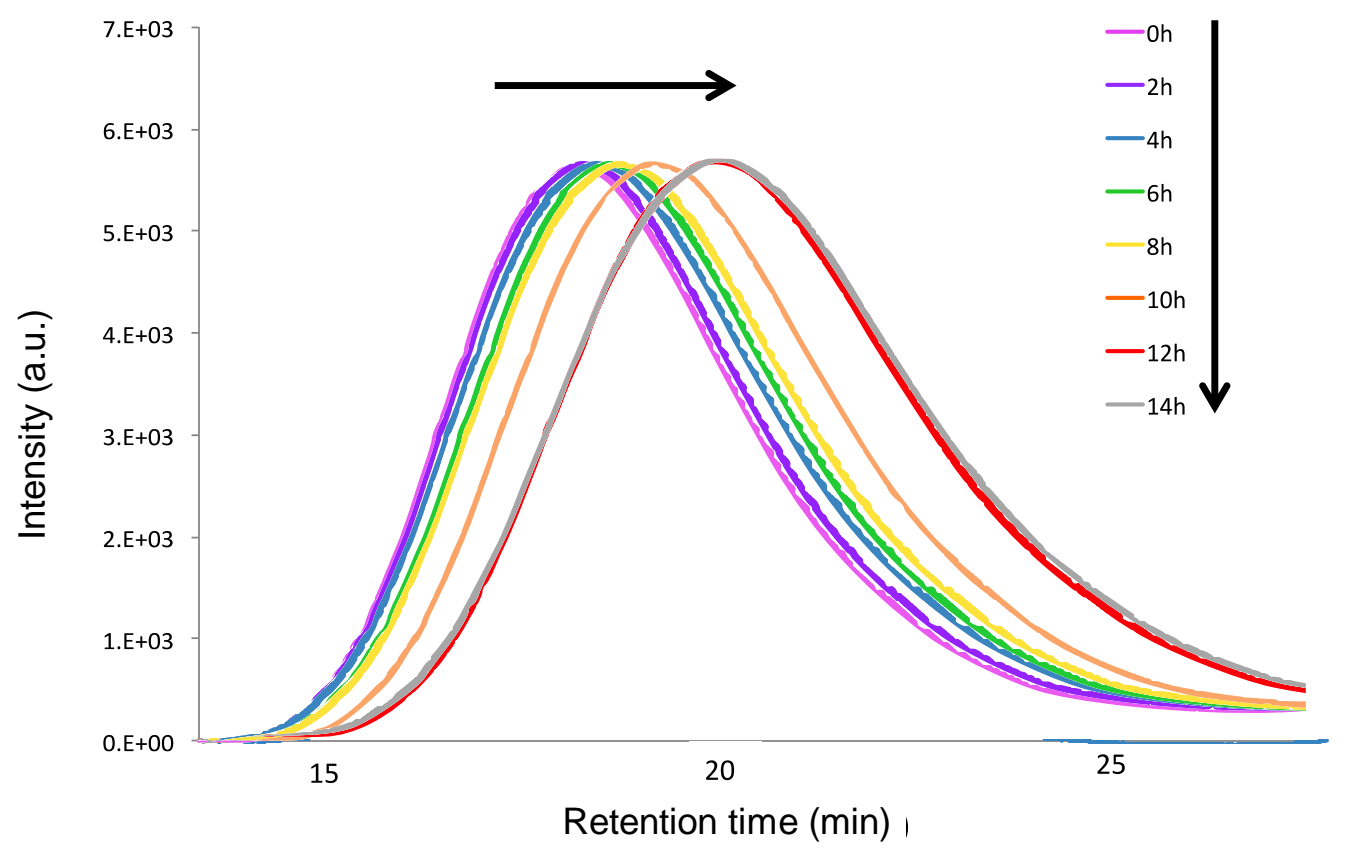

Fig. 5. Time-dependent SEC change of PEG-Graft PAN during the one-pot grafting reaction of PEG onto PAN using 1 at $160^{\circ} \mathrm{C}$ (eluent: DMF, $303 \mathrm{~K}$ ).

Finally, we investigated the catalyst-free thermal cross-linking of MKPs (Scheme 5). The cross-linking reactions of both PAN and NR with masked ketene moieties efficiently proceeded at $250{ }^{\circ} \mathrm{C}(1 \mathrm{~h})$ to give the respective cross-linked polymers as a solvent-insoluble part. In the case of cross-linked NR, the density of the network chain, cross-linking ratio, and efficiency of the cross-linking reaction were estimated by a modified Flory-Rehner equation [34], based on the swelling ratio of the insoluble polymer in toluene. As a result, it turned out that the interchain reaction efficiency of Meldrum's acid moieties on functionalized NR was $25 \%$. 

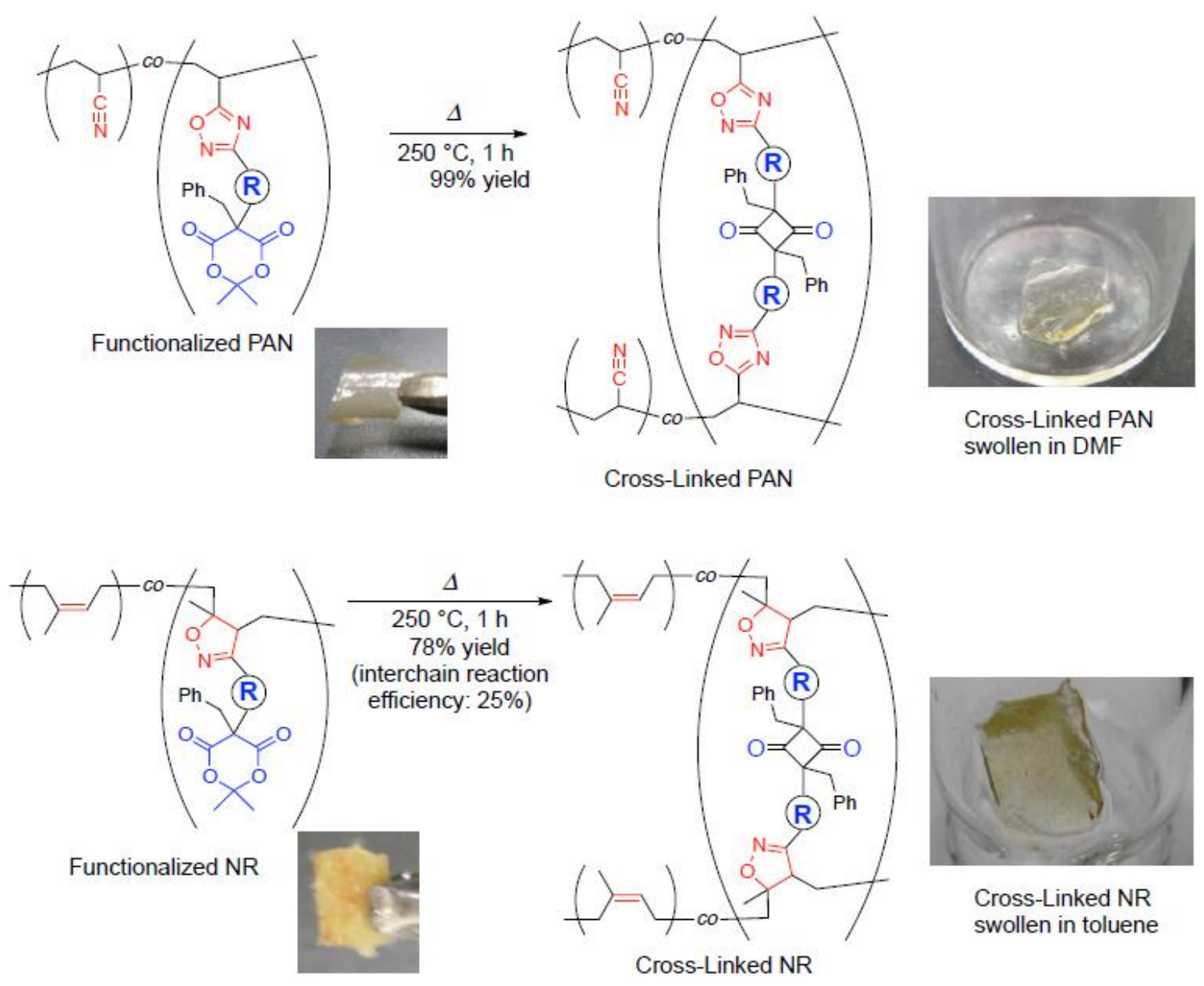

Scheme 5. Catalyst-free cross-linking of MKPs.

\section{Conclusion}

We have designed and developed a facile and widely applicable protocol for obtaining MKPs from unsaturated-bond-containing polymers via a catalyst-free click reaction and its subsequent modification to enable grafting and cross-linking. In this protocol, the cascade functionalization agent possessing both nitrile $\mathrm{N}$-oxide and masked ketene functionalities serves as a molecular glue to mediate the ligation reaction between unsaturated bonds and nucleophiles. The agent proved capable of catalyst-free click introduction of masked ketene moieties to the unsaturated bond-containing common polymers to give the MKPs. Heating of the resulting MKPs 
in the presence of nucleophiles afforded the corresponding adducts in high yields without a catalyst. On the other hand, heating of the MKPs in the absence of nucleophile resulted in the formation of cross-linked polymer based on the interchain dimerization reaction of Meldrum's acid moieties to give cyclobutadiones at the cross-linking points. The outcome would have broad applications not only to post-modification and surface modification of highly processed plastics made from common polymers but also supramolecular chemistry, because we have previously reported effective synthesis of rotaxane and polyrotaxane exploiting a cycloaddition of stable nitrile $N$-oxide [29-32]. $\quad$ Further invesitigations using the ambident agent along this line are currently underway.

\section{Acknowledgement}

The study was financially supported by a Grant-in-Aid for Scientific Research from the Ministry of Education, Culture, Sports, Science and Technology, Japan (No. 24685023).

\section{Appendix. Supplementary information.}

Supplementary information associated with this article can be found in the on-line version at http://www.journals.elsevier.com/polymer/. 


\section{References}

[1] Gil ES, Hudson SM. Prog Polym Sci 2004; 29(12): 1173-222.

[2] Mart RJ, Osborne RD, Stevens MM, Ulijn RV. Soft Matter 2006; 2(10): 822-35.

[3] Rapoport N. Prog Polym Sci 2007; 32(8-9): 962-90.

[4] Mendes PM. Chem Soc Rev 2008; 37(11): 2512-29.

[5] Mano JF. Adv Eng Mater 2008; 10(6): 515-27.

[6] Ganta S, Devalapally H, Shahiwala A, Amiji M. J Control Release 2008; 126(3): $187-204$.

[7] Ahn S, Kasi R M, Kim SC, Sharma N, Zhou Y. Soft Matter 2008; 4(6): 1151-57.

[8] Li MH, Keller P. Soft Matter 2009; 5(5): 927-37.

[9] Motornov M, Roiter Y, Tokarev I, Minko S. Prog Polym Sci 2010; 35 (1-2): $174-211$.

[10] Roy D, Cambre JN, Sumerlin BS. Prog Polym Sci 2010; 35(1-2): 278-301.

[11] Brown RFC, Eastwood FW, Harrington KJ. Aust J Chem 1974; 27(11): 2373-84.

[12] Baxter GJ, Brown RFC, Eastwood FW, Harrington KJ. Tetrahedron Lett 1975; 16(48): 4283-84.

[13] Soltani O, De Brabander JK. Angew Chem Int Ed 2005; 44(11): 1696-99.

[14] For a selected review, see: Tidwell T. Ketenes II, Second Edition, John Wiley \& Sons, Inc. New Jersey 2006.

[15] Sudo A, Uchino S, Endo T. Macromolecules 1999; 32(5): 1711-3.

[16] Nagai D, Sudo A, Endo T. Macromolecules 2006; 39(26): 8898-90.

[17] For a selected review, see: Hyatt JA, Reynolds PW. Org React 1994; 45: 159-646.

[18] Kumbaraci V, Talinli N, Yağci Y. Macromol Rapid Commun 2007; 28(1): 72-7.

[19] Tasdelen MA, Kumbaraci V, Talinli N, Yağci Y. Macromolecules 2007; 40(13): $4406-8$. 
[20] Durmaz YY, Kumbaraci V, Demirel AL, Talinli N, Yağci Y. Macromolecules 2009; 42(11): 3743-49.

[21] Leibfarth FA, Kang M, Ham M, Kim J, Campos LM, Gupta N, Moon B, Hawker CJ. Nat Chem 2010; 2(3): 207-12.

[22] Koyama Y, Miura K, Cheawchan S, Seo A, Takata T. Chem Commun 2012; 48 (83): 10304-6.

[23] Koyama Y, Yonekawa M, Takata T. Chem Lett 2008; 37(12): 918-9.

[24] Lee YG, Koyama Y, Yonekawa M, Takata T. Macromolecules 2009; 42(20): 7709-17.

[25] Lee YG, Yonekawa M, Koyama Y, Takata T. Chem Lett 2010; 39(5): 420-1.

[26] Koyama Y, Seo A, Takata T. Nippon Gomu Kyokaishi 2011; 84: 111-6.

[27] Koyama Y, Takata T. Kobunshi Ronbunshu 2011; 68(4): 147-59.

[28] Yonekawa M, Koyama Y, Kuwata S, Takata T. Org Lett 2012; 14(4): 1164-67.

[29] Matsumura T, Ishiwari F, Koyama Y, Takata T. Org Lett 2010; 12(17): 3828-31.

[30] Lee YG, Koyama Y, Yonekawa M. Takata T. Macromolecules 2010; 43(9): 4070-80.

[31] Jang K, Miura K, Koyama Y, Takata T. Org Lett 2012; 14(12): 3088-91.

[32] Iguchi H, Uchida S, Koyama Y, Takata T. ACS Macro Lett. 2013; 2: 527-30.

[33] Desai UV, Pore DM, Mane RB, Solabannavar SB, Wadgaonkar PP. Synth Commun 2004; 34(1): 25-32.

[34] Campbell DS. J Appl Polym Sci 1970; 14(6): 1409-19.

[35] See, supplementary information. 


\section{Captions for Schemes}

Scheme 1. Synthetic pathway of 1.

Scheme 2. Catalyst-free ligation between unsaturated bond and nucleophile using $\mathbf{1}$.

Scheme 3. Catalyst-free functionalization of unsaturated-bond-containing polymers using 1.

Scheme 4. (i) Catalyst-free grafting of PEG $\left(\begin{array}{llll}M_{\mathrm{n}} & 350\end{array}\right)$ onto masked-ketene-functionalized EPDM and (ii) one-pot grafting of PEG onto PAN.

Scheme 5. Catalyst-free cross-linking of MKPs.

\section{Captions for Figures}

Fig. 1. Synthetic strategy for MKP and its subsequent modification to enable grafting and cross-linking using an ambident agent having nitrile $N$-oxide and masked ketene functionalities.

Fig. 2. ${ }^{1} \mathrm{H} \mathrm{NMR}$ spectra of (a) 1, (b) 3, and (c) 5 (400 MHz, $\mathrm{CDCl}_{3}, 298 \mathrm{~K}$ ).

Fig. 3. ${ }^{1} \mathrm{H}$ NMR spectra of (a) PAN, (b) functionalized PAN with $3 \%$ functionalization ratio, and (c) PEG-grafted PAN (400 MHz, DMSO- $d_{6}, 298 \mathrm{~K}$ ).

Fig. 4. SEC profiles of EPDM (black line) and PEG-grafted EPDM (red line) (eluent: DMF, $303 \mathrm{~K})$.

Fig. 5. Time-dependent SEC change of PEG-Graft PAN during the one-pot grafting reaction of PEG onto PAN using 1 at $160{ }^{\circ} \mathrm{C}$ (eluent: DMF, $303 \mathrm{~K}$ ). 


\section{Graphical abstract}

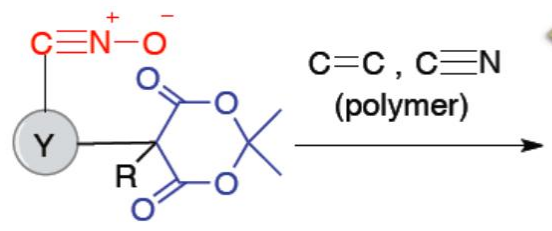

Amibident Agent with Nitrile $\mathrm{N}$-oxide and Masked ketene functionalities

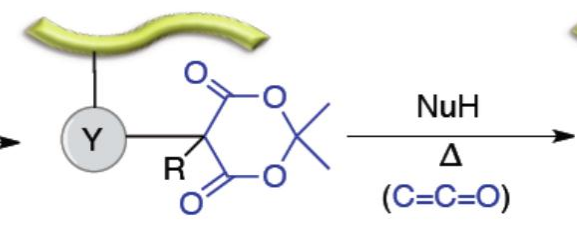

Masked-ketenefunctionalized polymer

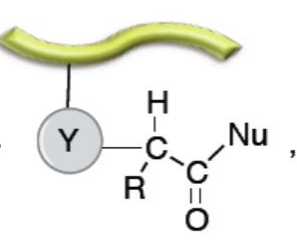

Grafted polymer

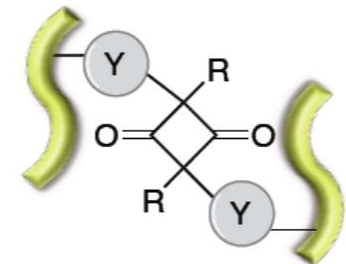

Cross-linked polymer 